\title{
Stimulus-Locked Responses on Human Upper Limb Muscles and Corrective Reaches Are Preferentially Evoked by Low Spatial Frequencies
}

\author{
Rebecca A. Kozak, ${ }^{1,5}$ Philipp Kreyenmeier, ${ }^{2}{ }^{-}$Chao Gu, ${ }^{3,5}{ }^{-}$Kevin Johnston,, ${ }^{4,5}$ and ${ }^{-}$Brian D. \\ Corneil ${ }^{1,3,4,5}$
}

https://doi.org/10.1523/ENEURO.0301-19.2019

\begin{abstract}
${ }^{1}$ Graduate Program in Neuroscience, Western University, London, Ontario N6A 5B7, Canada, ${ }^{2}$ Graduate Program in Neuro-Cognitive Psychology, Ludwig Maximilian University of Munich, Munich 80539, Germany, ${ }^{3}$ Department of Psychology, Western University, London, Ontario N6A 5B7, Canada, ${ }^{4}$ Department of Physiology and Pharmacology, Western University, London, Ontario N6A 5B7, Canada, and ${ }^{5}$ Robarts Research Institute, London, Ontario N6A 5B7, Canada
\end{abstract}

\begin{abstract}
In situations requiring immediate action, humans can generate visually-guided responses at remarkably short latencies. Here, to better understand the visual attributes that best evoke such rapid responses, we recorded upper limb muscle activity while participants performed visually-guided reaches towards Gabor patches composed of differing spatial frequencies (SFs). We studied reaches initiated from a stable posture (experiment 1, a static condition), or during on-line reach corrections to an abruptly displaced target (experiment 2 , a dynamic condition). In both experiments, we detail the latency and prevalence of stimulus-locked responses (SLRs), which are brief bursts of EMG activity that are time-locked to target presentation rather than movement onset. SLRs represent the first wave of EMG recruitment influenced by target presentation, and enable quantification of rapid visuomotor transformations. In both experiments, reach targets composed of low SFs elicited the shortest latency and most prevalent SLRs, with SLR latency increasing and SLR prevalence decreasing for reach targets composed of progressively higher SFs. SLRs could be evoked in either the static or dynamic condition, and when present in experiment 2, were associated with shorter latency and larger magnitude corrections. The results in experiment 2 are consistent with a linkage between the forces produced by SLRs and the earliest portion of on-line reach corrections. Overall, our results demonstrate that stimuli composed of low SFs preferentially evoke the most rapid visuomotor responses that, in the context of rapidly correcting an on-going reaching movement, are associated with earlier and larger on-line reach corrections.
\end{abstract}

Key words: EMG; on-line correction; spatial frequency; visually guided reaching

\section{Significance Statement}

Humans have a remarkable capacity to respond quickly to changes in our visual environment. Although our visual world is composed of a range of spatial frequencies (SFs), surprisingly little is known about which frequencies preferentially evoke rapid reaching responses. Here, we systematically varied the SF of peripheral reach targets while measuring EMG activity on an upper limb muscle. We found that visual stimuli composed of low SFs elicit the most rapid and robust EMG responses, and also elicit corrective reaches at shorter latencies. Thus, when time is of the essence, low SFs preferentially drive fast visuomotor responses. 


\section{Introduction}

To reach toward a visible target, visual information is ultimately transformed into motor commands. Doing so requires extraction of visual attributes such as the color, shape, and size of a visible target, and integration of these features into a command that is relayed to the motor periphery typically via the corticospinal tract. However, there are instances where we have to respond as quickly as possible, for example, to catch a falling mug or to volley a tennis ball that deflects off the net during a tennis match. Which features of a visual stimulus best engender such rapid visuomotor responses?

One way to study this question in the laboratory is to examine the latencies at which participants respond to targets that are suddenly displaced during an on-going reaching movement. In such dynamic scenarios, humans can initiate on-line reach corrections within a remarkably short latency of $\sim 125 \mathrm{~ms}$ following the target displacement (Soechting and Lacquaniti, 1983; Day and Lyon, 2000). Veerman et al. (2008) systematically investigated the influence of visual attributes in on-line reach corrections, and reported that earlier responses were driven by stimuli defined by luminance, orientation, and size, but not other attributes such as color. These results demonstrate that some visual attributes, but not others, are crucial for these rapid on-line reach corrections.

Recently, we have described a novel way of investigating rapid visuomotor responses that can be studied from a static posture. Stimulus-locked responses (SLRs) are short-latency bursts of directionally-tuned EMG activity that evolve time-locked within $\sim 100 \mathrm{~ms}$ of stimulus onset (Pruszynski et al., 2010), persisting even if an eventual reach is withheld (Wood et al., 2015; Atsma et al., 2018). Although never studied in the same paradigm, there are many similarities in the response properties of SLRs and on-line corrections. For example, both SLRs (Gu et al., 2016) and the trajectory of the initial phase of on-line corrections (Day and Lyon, 2000) are inexorably drawn toward the visual target, even when participants are instructed to move in the opposite direction. We and others have hypothesized that SLRs, and by extension the initial phase of an on-line reach corrections, are the product of

Received July 31, 2019; accepted August 28, 2019; First published September 5, 2019.

The authors declare no competing financial interests.

Author contributions: R.A.K., K.J., and B.D.C. designed research; R.A.K., P.K., and C.G. performed research; R.A.K., P.K., C.G., and B.D.C. analyzed data; R.A.K. and B.D.C. wrote the paper.

This work was supported by the Gouvernement du Canada Natural Sciences and Engineering Research Council of Canada (NSERC) Discovery Grant RGPIN 311680 and by the Canadian Institutes of Health Research Operating Grant MOP-93796. R.A.K. was supported by an Ontario Graduate Scholarship. C.G. was supported by an NSERC Canada Graduate Doctoral Scholarship.

Acknowledgements: We thank Dr. Andrew Pruszynski for the use of his exoskeleton and Rodrigo Maeda for assistance in the initial setup.

Correspondence should be addressed to Brian D. Corneil at bcorneil@uwo.ca.

https://doi.org/10.1523/ENEURO.0301-19.2019

Copyright (C) 2019 Kozak et al.

This is an open-access article distributed under the terms of the Creative Commons Attribution 4.0 International license, which permits unrestricted use, distribution and reproduction in any medium provided that the original work is properly attributed. a tecto-reticulospinal pathway that is mediated through the intermediate and deep layers of the superior colliculus (SCi; Pruszynski et al., 2010; Corneil and Munoz, 2014). Consistent with this, SLRs (Wood et al., 2015), on-line reach corrections (Veerman et al., 2008), and visual responses within the SCi (Marino et al., 2012) are all evoked at a shorter latency by high contrast stimuli.

The current study was motivated by a recent paper which reported that visual responses in the SCi are dependent on the spatial frequency (SF) of a visual stimulus, with SCi neurons responding sooner to lower SFs (Chen et al., 2018). Our hypothesis that the visual response in the SCi drives both the SLR and the earliest component of on-line reach corrections predicts that both SLRs and on-line reach corrections should evolve sooner for lower SF stimuli. We test this prediction in two experimental frameworks, measuring SLRs alone in reaches starting from a static posture (experiment 1), and measuring both SLRs and on-line reach corrections in a dynamic task where a reach target is displaced in mid-flight (experiment 2). Consistent with our hypothesis, we find that rapid visuomotor responses, whether indexed by SLRs or the latency of on-line reach corrections, are preferentially evoked by stimuli composed of low SFs.

\section{Materials and Methods}

\section{Participants}

A total of 33 participants (18 females, 15 males; mean age: 23.9 years, SD: 3.4) completed at least one of two experiments. All participants provided informed consent, were paid for their participation, and were free to withdrawal from the experiment at any time. All but two participants were right-handed. All participants had normal or corrected-to-normal vision, with no current visual, neurologic, or musculoskeletal disorders. All procedures were approved by the Health Science Research Ethics Board at the University of Western Ontario.

\section{Apparatus}

Participants were seated in a KINARM robotic exoskeleton (BKIN Technologies), and performed reaching movements with their right arm (Fig. 1A). As previously described (Scott, 1999; Pruszynski et al., 2008), this robot permits arm movements in a horizontal plane via shoulder and elbow flexion and extension, and allows for torque application at these joints. Visual stimuli were projected from a downward facing LED monitor (LG 47LS35A, size: 47" resolution: $1920 \times 1080$ pixels), onto an upward facing mirror. A shield underneath the mirror occluded direct vision of the hand, but real-time hand position was represented on the monitor via a black dot with a diameter of $1 \mathrm{~cm}$. A photodiode was used to indicate the precise time of onset of the peripheral visual stimulus, and all kinematic and EMG data were aligned to photodiode onset. Eye movements were not measured.

\section{Experimental design \\ Experiment 1a: static task}

Participants $(n=16)$ performed visually guided reaches (Fig. 1A,B) toward peripheral stimuli composed of stationary Gabor patches (see Composition of peripheral Gabor 
A

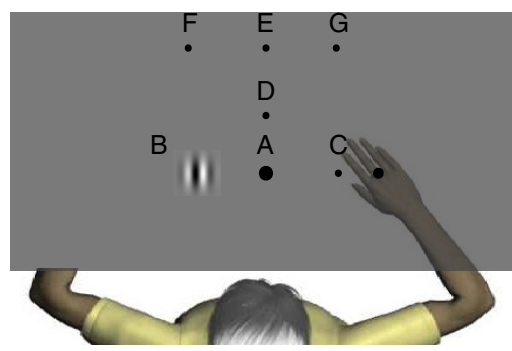

B

\begin{tabular}{|c|c|c|c|c|c|}
\hline EXPERIMENT & $\begin{array}{l}\text { START } \\
\text { HAND }\end{array}$ & $\begin{array}{c}\text { START } \\
\text { EYE }\end{array}$ & $\begin{array}{c}\text { POTENTIAL } \\
\text { TARGETS }\end{array}$ & $\begin{array}{l}\text { GO } \\
\text { CUE }\end{array}$ & $\begin{array}{c}\text { SPATIAL } \\
\text { FREQUENCIES }\end{array}$ \\
\hline 1A & A & A & $50 \%$ B, $50 \%$ C & $\begin{array}{l}\text { Target } \\
\text { Onset }\end{array}$ & $\begin{array}{c}.15, .3, .6,1.11 \\
1.60,2.22 \mathrm{cpd}\end{array}$ \\
\hline $1 B$ & D & $E$ & $50 \%$ F, $50 \%$ G & $\begin{array}{l}\text { Target } \\
\text { Onset }\end{array}$ & $\begin{array}{c}.56,2.22,4.44 \\
\mathrm{cpd}\end{array}$ \\
\hline 2 & A & $E$ & $\begin{array}{c}33 \% \mathrm{E} ; 33 \% \\
\mathrm{~F} ; 33 \% \mathrm{G}\end{array}$ & $\begin{array}{c}\mathrm{E} \\
\text { Onset }\end{array}$ & $\begin{array}{c}.56,2.22,4.44 \\
\mathrm{cpd}\end{array}$ \\
\hline
\end{tabular}

C PEC muscle activity $(\mu \mathrm{V})$

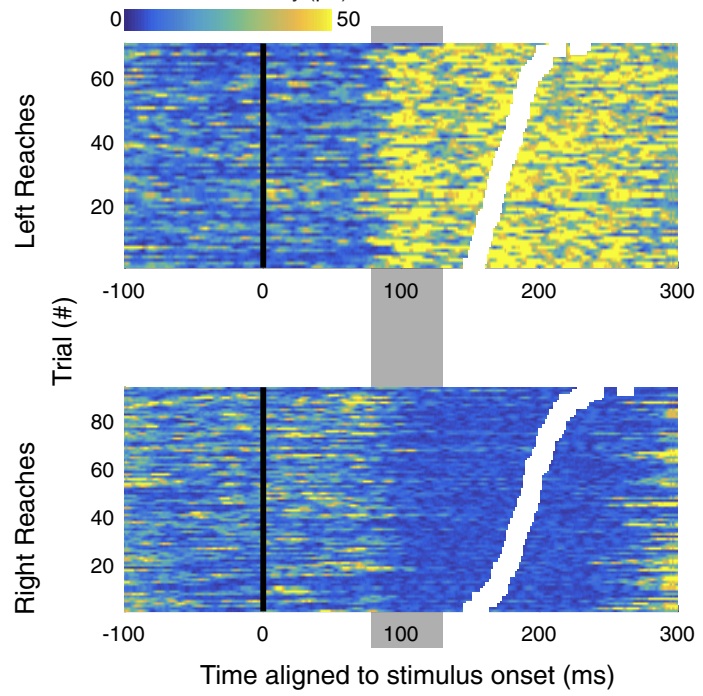

D

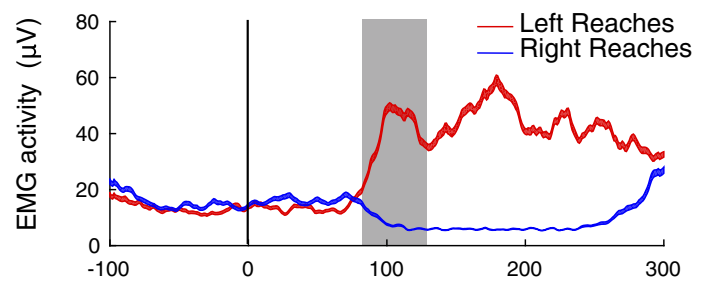

E

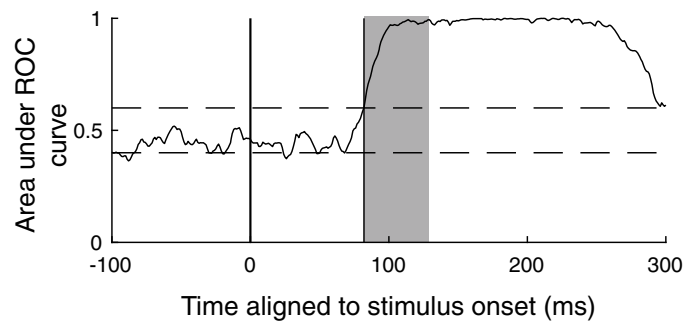

Figure 1. Experimental design and SLRs. A, B, General display and configuration of targets $(\boldsymbol{A})$ and corresponding hand and eye start positions, potential targets, go cues, and SFs used for each of the experiments (B; see Materials and Methods for more details). $\boldsymbol{C}$, Trial-by-trial recruitment of right PEC for an example subject (s30, from experiment 2) generating left or right reaches. Each row is a different trial, with color conveying degree of recruitment. Trials sorted by RT (white boxes), and aligned to stimulus onset. The SLR (highlighted in gray vertical box) appears as a vertical banding of increased or decreased recruitment aligned to left or right stimulus presentation, respectively, rather than movement onset. $\boldsymbol{D}, \boldsymbol{E}$, Mean EMG activity ( \pm SE; $\boldsymbol{D})$ and time-series ROC analysis $(\boldsymbol{E})$ for data shown in $\boldsymbol{C}$. Horizontal dashed lines in $\boldsymbol{E}$ shows the 0.6 or 0.4 level which constitutes discrimination threshold.

target patches below), starting from a static posture in which the hand was not in motion. Throughout the experiment, a constant torque of $2 \mathrm{Nm}$ was applied to the shoulder for the entire experiment, increasing tonic activity on the muscle of interest. Doing so allowed for observations in decreases of muscle recruitment when the arm moved in the non-preferred direction for the muscle of interest. Participants initiated each trial by bringing their hand into a central black stimulus, which was located 45 $\mathrm{cm}$ in front of them relative to their midline. This central stimulus had a $1 \mathrm{~cm}$ diameter and changed to white once the hand attained this position. Participants were instructed to foveate this start position before target onset. The trial was reset if the hand exited the start position before completion of a hold period (randomized from 1 to $1.5 \mathrm{~s})$. Simultaneous with the disappearance of the start position stimulus, a Gabor patch subtending $7 \mathrm{~cm}$ then appeared either $10 \mathrm{~cm}$ to the left or right of the start position; the center of this patch was at an eccentricity of $\sim 9^{\circ}$ to the left or right of the start position; subtending $\sim 12^{\circ}$. The Gabor patch was composed of one of six SFs ranging between 0.15 and 2.22 cycles per degree (cpd;
Fig. 1B; see Composition of peripheral Gabor target patches below for details on how Gabor patches were constructed). Our lowest SF was implemented as a control target, as we presented a very low SF, effectively presented as a Gaussian blurred black dot. Participants were instructed to reach toward the peripheral target as quickly as possible, with the trial ending when the virtual cursor made contact with the Gabor. There were 12 unique trial conditions (six SFs, each presented to the left and right). Participants completed five blocks of 240 trials each, with each block containing 20 repetitions of each unique trial condition presented pseudorandomly without replacement, yielding a total of 100 trials for each unique trial condition.

\section{Experiment 1b: static task with higher SF targets}

In experiment $1 \mathrm{~b}$, peripheral targets were placed at an increased distance from the subject, permitting the presentation of higher SFs than in experiment 1a. Stimuli were placed at locations resembling those used in experiment 2 (see Experiment 2: dynamic task below), so that the location of the targets relative to the eye and hand 
A
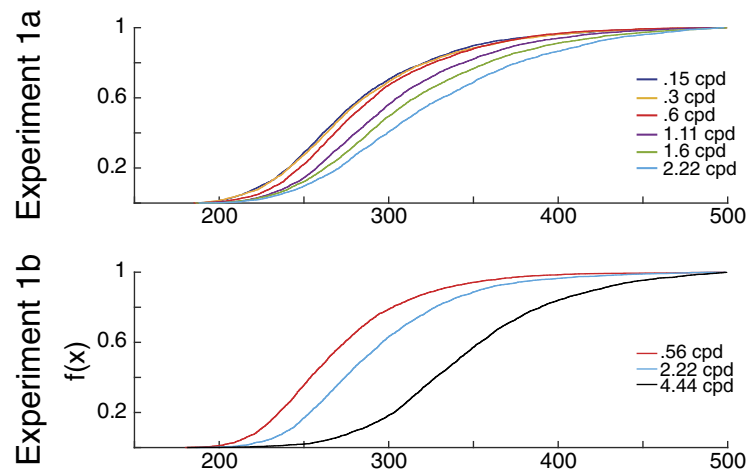

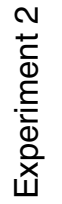

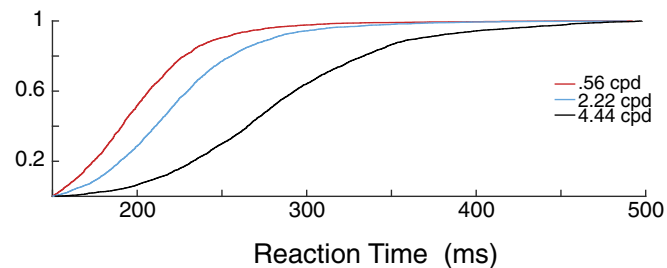

B
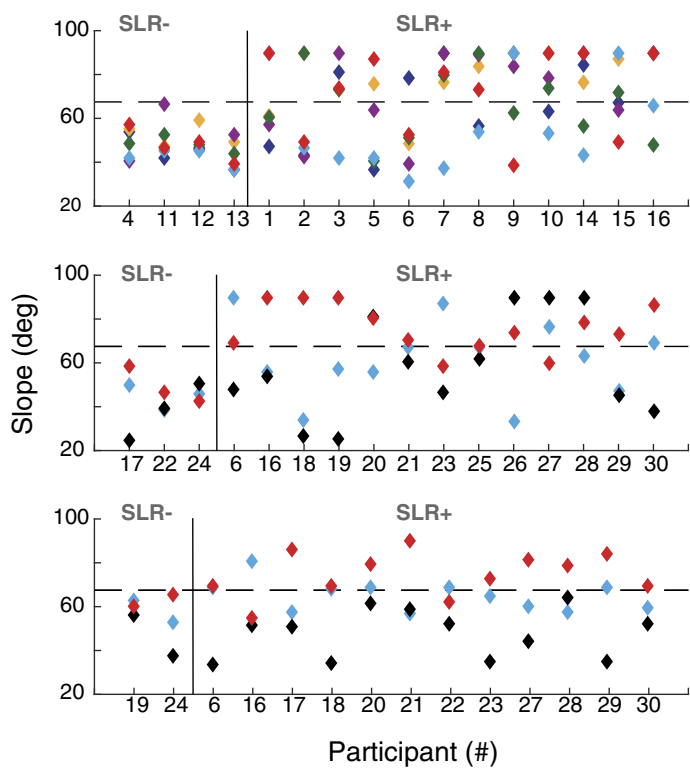

Figure 2. RTs and detection of SLRs. A, Cumulative RT distributions in all experiments, pooled across all participants and directions and segregated by SF. B. Slope of the relationship between discrimination time and average RT for early and late RT groups, for all experiments. Each data point represents a unique subject for a particular SF. Slopes are capped at $90^{\circ}$. Results are grouped into those who exhibiting an SLR in at least one condition (SLR+) or not (SLR-), given a slope threshold of $67.5^{\circ}$ (horizontal dashed line).

were approximately the same in experiments $1 \mathrm{~b}$ and 2 . A total of 16 participants, some of whom participated in experiment $1 \mathrm{a}$, also participated in experiment $1 \mathrm{~b}$ (Fig. $1 A, B) ; 14$ of 16 participants in experiment $1 \mathrm{~b}$ also participated in experiment 2 (for participant details, see Fig. 2B).

Participants initiated each trial by bringing their hand into a start position (2 cm diameter) located $55.5 \mathrm{~cm}$ in front of them. A constant torque of $3 \mathrm{Nm}$ was applied to the shoulder for the entire experiment. A higher torque was used than in experiment $1 \mathrm{a}$, as target positions were less in the preferred direction of the muscle. A fixation cross then appeared $10 \mathrm{~cm}$ above the central hold marker, which participants were instructed to look at while not moving their hand. Participants were required to maintain the initial hand position for a randomized period of 1-2 s. After this, a 5-cm diameter Gabor patch composed of one of three frequencies $(0.56,2.22,4.44 \mathrm{cpd}$; Fig. $1 B$ ) subtending $\sim 4.5^{\circ}$ of visual angle, was presented. The center of this patch was $\sim 8^{\circ}$ of visual angle to the left or right of the start position. Participants were instructed to reach and look to this peripheral target as quickly as possible. Participants completed four blocks of 150 trials each, with each block composed of 25 trials of each unique trial condition (three SFs to the right and left), yielding a total of 100 repeats of each unique trial condition.

\section{Experiment 2: dynamic task}

In experiment 2, participants ( $n=14$, all of whom also completed experiment $1 \mathrm{~b}$ in the same session) occasionally generated on-line corrections to stimuli that suddenly jumped to the left or right just after the start of a reaching movement. We term this a dynamic task, as the hand was in motion when the target stimulus was displaced, necessitating an on-line correction. Peripheral stimuli were presented at the same screen locations as in experiment $1 \mathrm{~b}$, and initial eye and hand positions were also dissociated. A constant torque was not applied during experiment 2, as participants in pilot experiments found this load to be too difficult to maintain for the duration of experiment 2.

Participants initiated each trial by first bringing their hand into a central hold position ( $38 \mathrm{~cm}$ in front of them), while they looked at a central fixation cross located 17.5 $\mathrm{cm}$ above the central hold stimulus (Fig. $1 A, B$ ). The central hold stimulus for the hand was placed closer to the participant in experiment 2 than in experiment $1 \mathrm{~b}$, as a longer reaching movement permitted more time for a mid-flight correction. Further, this configuration meant that the midflight hand position at peripheral target presentation closely resembled that used in experiment $1 \mathrm{~b}$. Participants then maintained this dissociated hand and eye position for 1-2 s. After this, the central fixation cross changed to a Gaussian blurred black dot, cueing the participant to reach toward this central location. All trial types were identical up to this time. On $1 / 3$ rd of all trials (termed control trials), no other event occurred and participants were simply required to reach toward the Gaussian blurred black dot. On the remaining 2/3rds of all trials (termed jump trials), the blurred Gaussian black dot was replaced by a peripheral target identical to those used in experiment $1 \mathrm{~b}$. The presentation of this peripheral target occurred when the hand exited the central hold position (1 $\mathrm{cm}$ in diameter), requiring participants to adjust an ongoing reach movement to either the left or right. Participants were instructed to reach as quickly as possible to 
the fixation target on control trials, and to try to correct their reach movements as quickly as possible on jump trials, so that they could move through the bottom aspect of the peripheral Gabor patch. There was a total of seven unique trial combinations: a control trial, and six different jump trials (three SFs per direction). Participants completed four blocks of 225 trials each, with each block composed pseudorandomly of 75 control trials and 25 repeats of each unique jump trial condition; thus, there were a total of 300 control trials and 100 trials of each unique jump trial condition.

\section{Composition of peripheral Gabor target patches}

All stimuli were created in MATLAB using the Psychophysics toolbox (Brainard, 1997; Pelli, 1997). Due to monitor resolution and the viewing distance of the targets, we were limited to an upper limit of $2.22 \mathrm{cpd}$ in experiment $1 \mathrm{a}$, and $4.44 \mathrm{cpd}$ in experiments $1 \mathrm{~b}$ and 2 . Stimuli consisted of vertical sinewaves overlaid with a Gaussian window. In all experiments, peripheral target stimuli consisted of perceptually contrast matched Gabor patches varying in SF measured in cpd of visual angle. Our motivation to implement a contrast matching procedure was two-fold. First, we performed this procedure in an attempt to isolate the effects of SF from perceived contrast, as contrast influences SLR latency and magnitude (Wood et al., 2015). Second, perceptual contrast matching mitigates some of the increases in RT with higher SFs (Breitmeyer, 1975), which was important given that larger magnitude SLRs precede shorter RTs (Pruszynski et al., 2010; Gu et al., 2016). We used a modified contrast matching procedure with foveal stimuli as described in (Davidson, 1968). A double random interleaved procedure was used for each SF, on a linearized ( $\gamma$-corrected) screen (mean background luminance $42.78 \mathrm{~cd} / \mathrm{m}^{2}$ ). Participants were presented a fixation point, followed by a flashed Gabor patch (200 ms), followed by a pause of $500 \mathrm{~ms}$, followed by a second flashed Gabor patch (200 ms); participants were instructed to "indicate the stimulus with more contrast," by button press. In each experiment, we used a single standard stimulus presented at a suprathreshold $85 \%$ contrast from the middle SF used in each experiment, with the comparison stimulus from each of the SFs being composed of a Gabor patch presented at a higher or lower contrast, as determined by a staircase procedure. Our rationale for using suprathreshold contrast is based on the relationship between SLR detectability and contrast (Wood et al., 2015). After a reversal (participant changes response from increasing to decreasing contrast or vice versa), the step size was decreased. Each staircase procedure required ten reversals, and the contrast for a matched stimulus was taken as the mean contrast of the last five reversals. All participants completed this procedure for all SFs used in a given experiment, yielding a unique set of perceptually contrastmatched Gabors across a range of SFs for each subject, which were then used in the experiment.

\section{Data acquisition and analysis}

As previously described (Gu et al., 2018), surface EMG activity was recorded from the clavicular head of the right pectoralis muscle with double-differential surface electrodes (Delsys Inc. Bagnoli-8 system). We placed two electrodes on each participant, and chose the recording exhibiting the higher level of background activity. EMG signals were sampled at $1000 \mathrm{~Hz}$, amplified by 1000, and rectified off-line. We excluded some or all data from five participants (one from experiment 1a, two from both experiments $1 \mathrm{~b}$ and 2 , and two from only experiment 2) due to an absence or very low level of recruitment for movements in the preferred direction of the muscle. Recall as well that the data from experiments $1 \mathrm{~b}$ and 2 were collected from the same participants on the same day. Given that some analyses compare within-subject data across experiments $1 b$ and 2, we used data from the same recording electrode wherever possible. This was not possible in three participants, where one of the recording electrodes fell off between experiments 1 and 2 .

All kinematic data were sampled at $1000 \mathrm{~Hz}$ by the Kinarm data acquisition system. Reaction time (RT) was calculated as time from peripheral target appearance (measured by the photodiode) to the initiation of the reaching movement. In all experiments, reach initiation was determined using the lateral velocity toward left and right targets, extrapolating a line drawn between the crossings of $25 \%$ and $75 \%$ of single trial peak velocities back to zero (as in Veerman et al., 2008).

Our previous work on upper limb muscle recruitment during visually-guided reaching has distinguished the small burst of EMG activity aligned to stimulus onset (i.e., the SLR) from the larger, later burst of EMG activity associated with onset of the reaching movement (Gu et al., 2016, 2018). The distinction between the SLR and the later movement-related burst becomes blurred for shorter-RT movements. Given that the goal of this manuscript is to isolate the effects of the SF of a peripheral stimulus on the SLR, we excluded trials below a particular RT (excluding trials with RTs $<185 \mathrm{~ms}$ in experiments 1a and $1 \mathrm{~b}$, and trials with RTs $<150 \mathrm{~ms}$ in experiment 2), since RTs of on-line reach corrections tend to be shorter than RTs initiated from a static posture (excluding $0.2 \%$, $0.25 \%$, and $4.3 \%$ of all trials for experiments $1 \mathrm{a}, 1 \mathrm{~b}$, and 2 , respectively). In all experiments, we also excluded trials with RTs $>500 \mathrm{~ms}$, due to presumed inattentiveness (excluding $2.2 \%, 4.4 \%$, and $1.7 \%$ of all trials for experiments $1 \mathrm{a}, 1 \mathrm{~b}$, and 2 , respectively). Finally, in all experiments, we excluded trials where the hand deviated by $>2$ $\mathrm{cm}$ in the wrong direction $(<1 \%$ of all trials across all experiments).

We used a receiver-operating characteristic (ROC) analysis to quantitatively define the presence and latency of the SLR, as described previously (Corneil et al., 2004; Pruszynski et al., 2010). We separated EMG activity based on stimulus location and SF condition. EMG activity for the same SF but opposite stimulus locations was then analyzed at every time sample $(1 \mathrm{~ms})$ between 100 ms before and $300 \mathrm{~ms}$ after stimulus presentation. For each sample we calculated the area under the ROC curve, which indicates the probability that an ideal observer could discriminate between leftward and rightward stimulus presentations based solely on the EMG activity. A 
value of 0.5 indicates chance discrimination, whereas values of 1 or 0 indicate perfectly-correct or incorrect discrimination, respectively. Discrimination threshold was set to 0.6 , which corresponds approximately to the upper 95\% confidence interval determined using a bootstrapping procedure (Goonetilleke et al., 2015). ROC latency was defined as the time where the ROC time series surpassed threshold and remained above it for a minimum of eight out of ten samples.

A representation of trial-by-trial EMG activity, mean EMG, and the associated time-series ROC analysis that we use to define SLR characteristics is shown for a representative participant in Figure $1 C-E$. The SLR is visible in this example as a vertical banding indicating a change in muscle recruitment aligned to stimulus as opposed to movement onset (Fig. 1C), and as a transient increase or decrease in activity in mean EMG activity for leftward and rightward stimuli, respectively (Fig. $1 D$, highlighted by the gray boxes). To determine whether the initial change in EMG activity was more locked to stimulus onset than movement onset, for each SF we performed a mediansplit of trials based on RT and conducted separate ROC time-series analyses for both "early RT" (i.e., the subset of shorter RT trials) and "late RT" (the subset of longer RT trials) subsets (Goonetilleke et al., 2015; Wood et al., 2015). We then calculated the slope of the line connecting the average RT and the SLR latency for the "early" and "late" RT subgroups (for examples, see Fig. $3 C$ ). Participants were classified as exhibiting a SLR if the slope of the line exceeded $67.5^{\circ}$, which is halfway between a line parallel to the line of unity $\left(45^{\circ}\right)$ and vertical $\left(90^{\circ}\right)$; a slope $>67.5^{\circ}$ indicates that EMG recruitment was more locked to stimulus rather than movement onset. If a SLR was detected using the median split analysis, a subsequent time-series ROC plot was constructed using all trials to determine the SLR latency (the first of 8 of 10 consecutive points $>0.6$ ) and the SLR magnitude (defined as the area between leftward and rightward mean EMG traces calculated from interval spanning from the SLR latency to 15 ms later).

In experiment 2, we also conducted a time-series ROC analysis to determine the latency of on-line reach corrections, based on the lateral position of the manipulandum (position along the $x$-axis) toward leftward or rightward stimuli on jump trials. As with the analysis of EMG activity, the threshold was set to 0.6 , and the latency was determined as the first of 8 of 10 consecutive points exceeding this threshold. We also defined the magnitude of the reach correction as the area between the mean trajectories for left and right reach corrections in an interval spanning from the time of discrimination to $400 \mathrm{~ms}$ after stimulus onset. Thus, larger magnitudes are indicative of an increased ability to move laterally toward a target.

\section{Statistical analyses}

Statistical analyses were performed in MATLAB (version R2016a, The MathWorks, Inc.). Results were analyzed with paired $t$ tests or one-way repeated measures ANOVAs, unless otherwise stated, and post hoc tests were Bonferroni corrected where appropriate. $\chi^{2}$ tests were used to analyze changes in SLR prevalence across SF.

\section{Results}

\section{Higher SFs elicit longer RTs}

We first quantified the influence of the SF of a stimulus on RT, across all experiments and participants. Participants performed reaches from a static hand position to stimuli placed either right or left of a start position (experiment 1a; Fig. $1 A, B$ ), right-outward or left-outward of a start position (experiment $1 \mathrm{~b}$; Fig. $1 A, B$ ), or performed on-line reach corrections of an outward reach movement to targets displaced to the left or right (experiment 2; Fig. $1 A, B)$. As expected based on Breitmeyer (1975), and even with the perceptual contrast-matching procedure, in all experiments we found that the SF of a stimulus influenced RTs [one-way repeated measures ANOVA of mean RT; experiment 1a; $F_{(5,75)}=69.44, p=9.83^{\wedge}-27$ (Fig. $2 A$, top panel); experiment $1 \mathrm{~b} ; F_{(2,30)}=134.04, p=1.10^{\wedge}-15$ (Fig. $2 A$, middle panel); experiment $2 ; F_{(2,26)}=99.68, p=$ $6.42^{\wedge}-13$ (Fig. $2 A$, bottom panel)]. As shown in Figure $2 A$, RTs tended to increase for higher SFs (post hoc analyses via paired $t$ tests Bonferroni-corrected for multiple comparisons; the only insignificant comparison between adjacent SFs were between 0.15 vs $0.3 \mathrm{cpd}$ and 0.3 vs 0.6 cpd in experiment 1a). Importantly, RT distributions for a given SF and the next-highest SF often overlapped. In a subsequent analysis, we will exploit this overlap to mitigate the potential confound of RT on the SLR.

\section{Earlier SLRs are preferentially evoked by lower SFs}

Next, we examined the influence of the SF of a stimulus on SLR characteristics. As shown in previous work, the SLR on limb muscles consists of a brief increase or decrease in the recruitment of muscle activity $(\sim 100 \mathrm{~ms}$; Pruszynski et al., 2010). Figure $1 C$ shows one example of a prominent SLR in one representative subject from experiment 2, who generated leftward or rightward visuallyguided corrections to a 0.56 -cpd displaced target. In this example, the SLR appears as the prominent vertical band of EMG activity that begins $\sim 90 \mathrm{~ms}$ after visual target presentation, regardless of the ensuing RT (for other examples of SLRs, see Figs. 3, 4).

We used a time-series ROC analysis (see Materials and Methods) to detect the presence or absence of an SLR. A participant was deemed to exhibit an SLR at a particular $\mathrm{SF}$ if the slope of a line connecting the RT plotted as a function of the discrimination time for the early and late RT groups exceeded $67.5^{\circ}$ (indicating EMG activity is more locked to stimulus onset as opposed to movement onset). Using this analysis, we detected an SLR + observation in at least one SF condition in 12 out of 16 participants in experiment 1a (Fig. 2B, top panel; for data from a representative subject for experiment 1a, see Fig. 3), 11 out of 16 participants in experiment 1b (Fig. 2B, middle panel; for data from a representative subject for experiment $1 \mathrm{~b}$, see Fig. $4 A-C$ ), and 12 out of 14 participants in experiment 2 (Fig. 2B, bottom panel; for data from a representative subject for experiment 2, see Fig. $4 D-F$ ). The data shown in Figures 3, 4 also show a qualitative pattern 
A Left Reaches
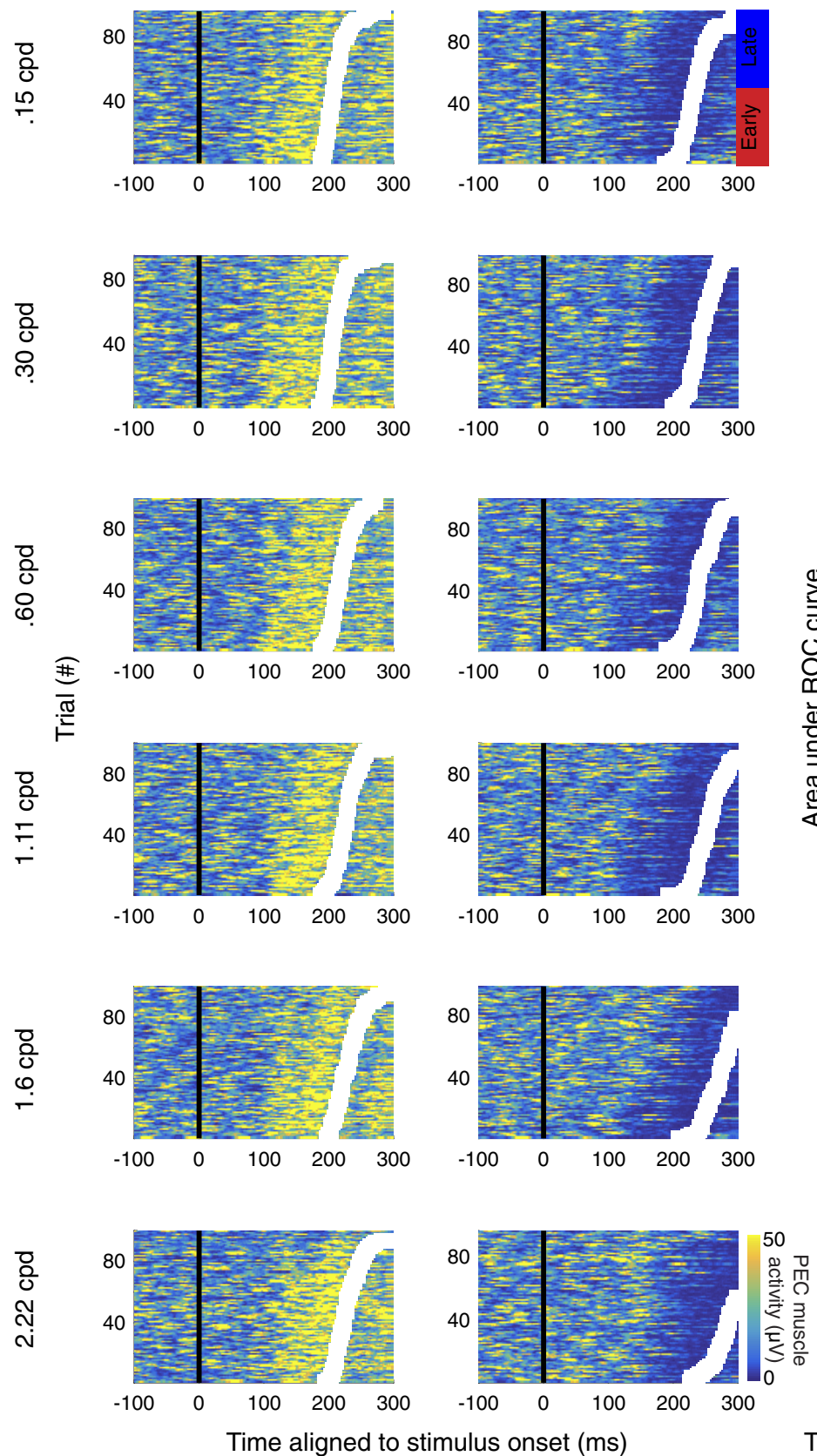

B
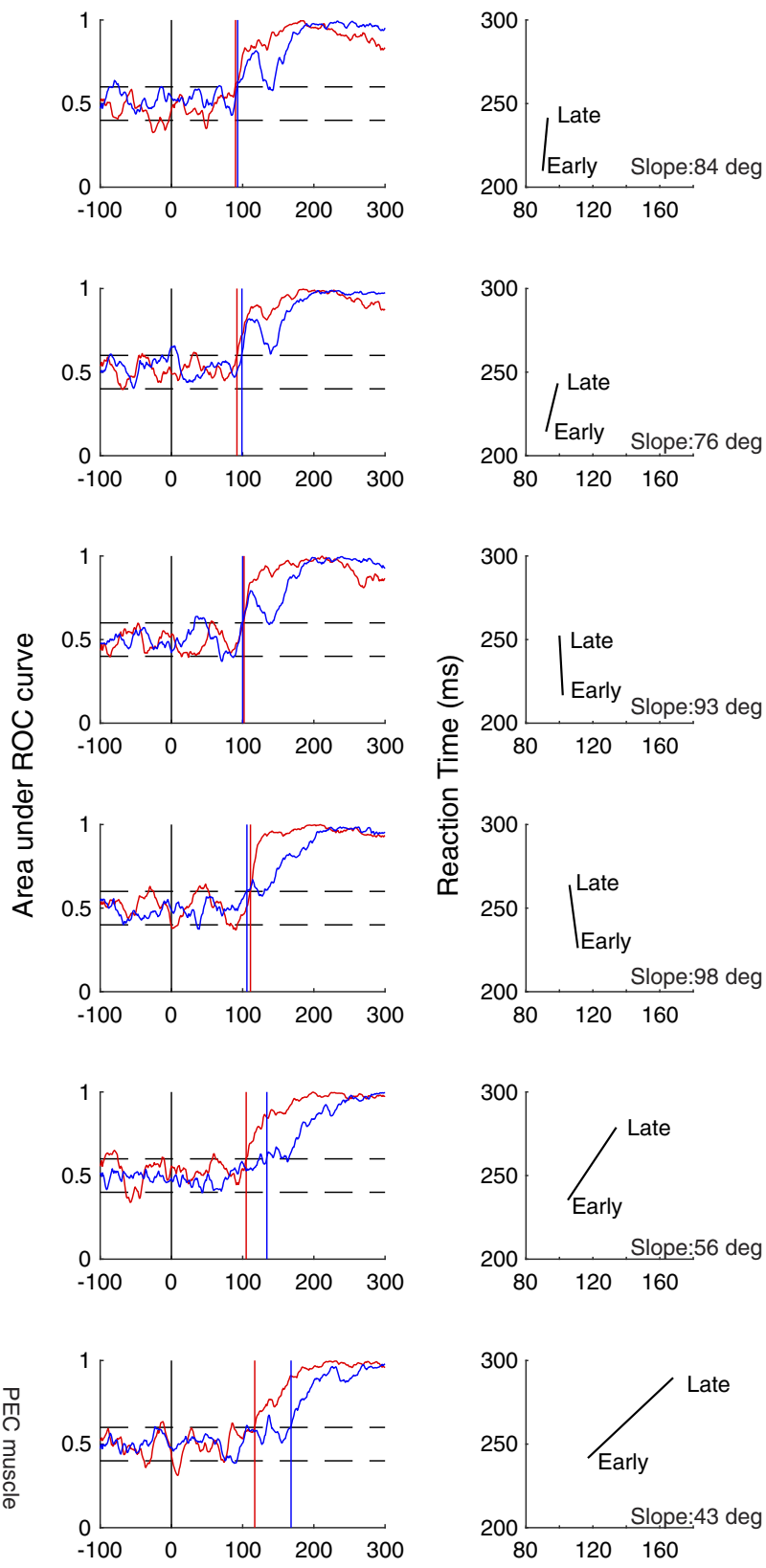

Time aligned to stimulus onset $(\mathrm{ms}) \quad$ Discrimination time $(\mathrm{ms})$

Figure 3. Example results from experiment 1a. A, Muscle recruitment from an example subject (s14) from experiment 1a, segregated by movement direction and SF. Same format as Figure 1C. Trials were separated into early (red) or late (blue) RT groups based on the median RT. B, Time-series ROC plot analysis from early (red) or late (blue) RT groups for data in $\boldsymbol{A}$. Vertical colored solid lines depict discrimination time. $\boldsymbol{C}$, Plot of the average RT versus discrimination time for early and late groups. The data were deemed to exhibit an SLR if the slope of the line exceeded $67.5^{\circ}$, which indicated that the initiation of EMG recruitment was more locked to stimulus rather than movement onset.

whereby lower SFs evoke shorter latency and more distinct SLRs when compared to SLRs evoked by higher SFs (e.g., 0.15 vs $1.6 \mathrm{cpd}$ rows in Fig. 3, 0.56 vs 2.22 cpd Fig. 4).

As described previously (Wood et al., 2015), SLRs can consist of a brief oscillation of increases and decreases in EMG activity if movement-related activity is delayed or absent (see also Atsma et al., 2018). Such oscillations are apparent in some of examples shown here, and produce a dip in the time-series ROC that can be particularly apparent for the late-RT groups in experiments $1 \mathrm{a}$ and $1 \mathrm{~b}$ (Fig. 3B, top three rows; Fig. $4 B$, top row). Importantly, such dips in the time-series ROC are less apparent in the short-RT groups and in experiment 2, which we surmise relates to movement-related activity arriving near the end or shortly after the SLR interval. 


\section{Experiment 1b}

A Left Reaches
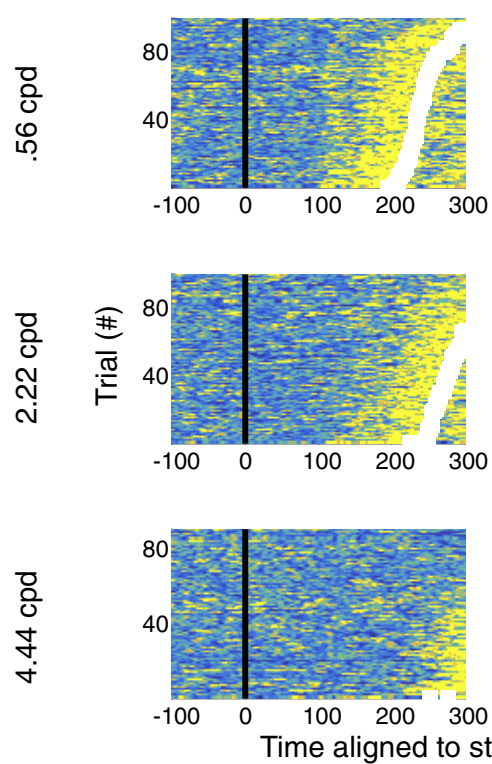

Right Reaches
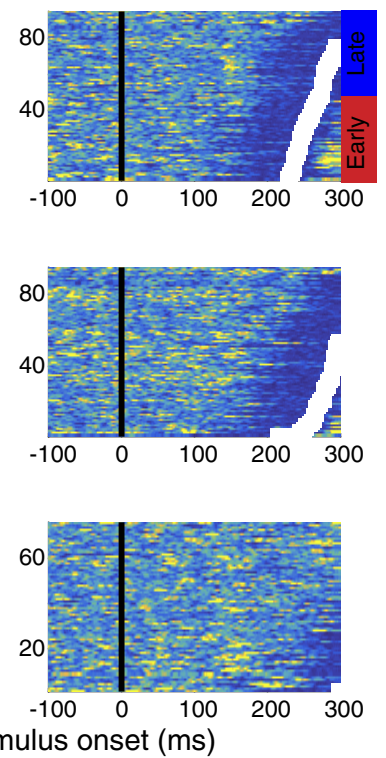

B
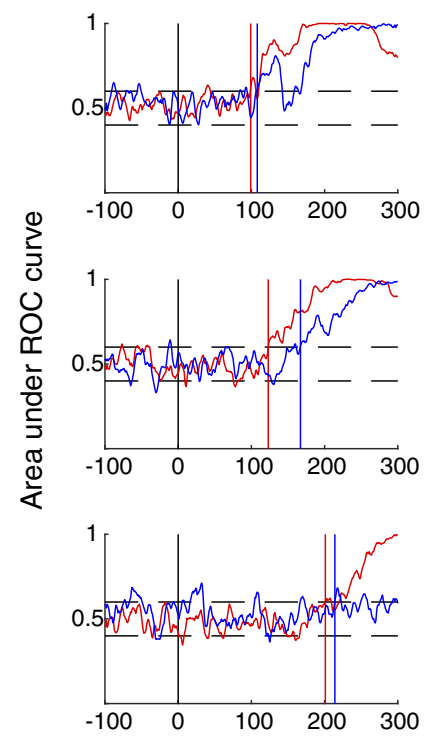

Time aligned to stimulus onset (m
C
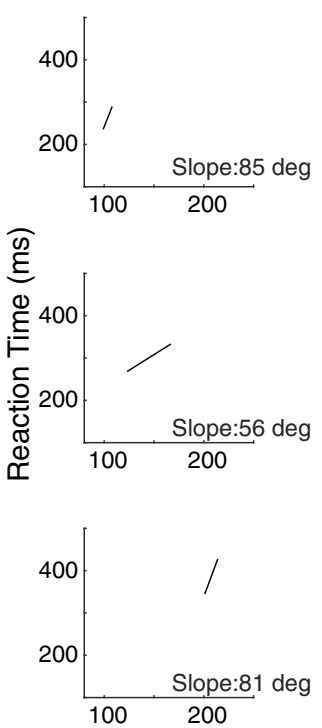

s) Discrimination time (ms)

\section{Experiment 2}

\section{D}
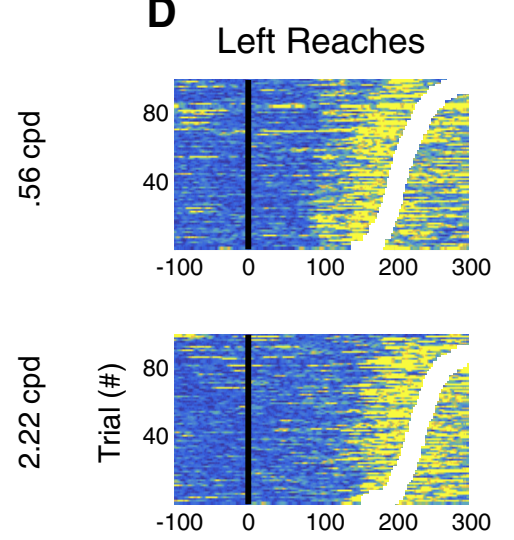

8
8
8
$\dot{y}$
$\dot{7}$

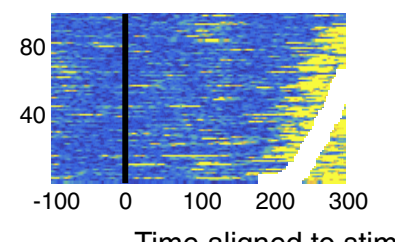

Right Reaches
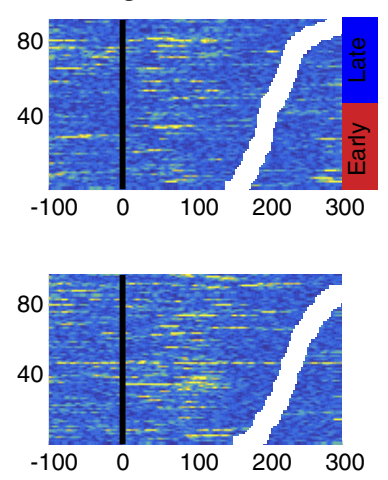

E
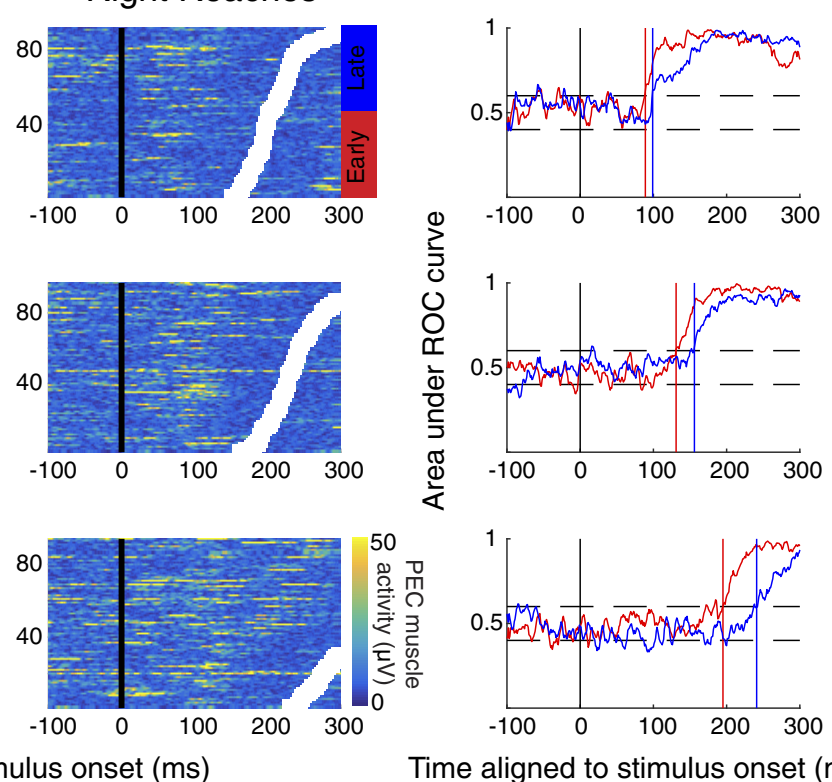

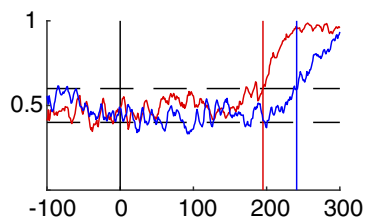

Time aligned to stimulus onset $(\mathrm{ms})$ Discrimination time (ms)
$\mathbf{F}$
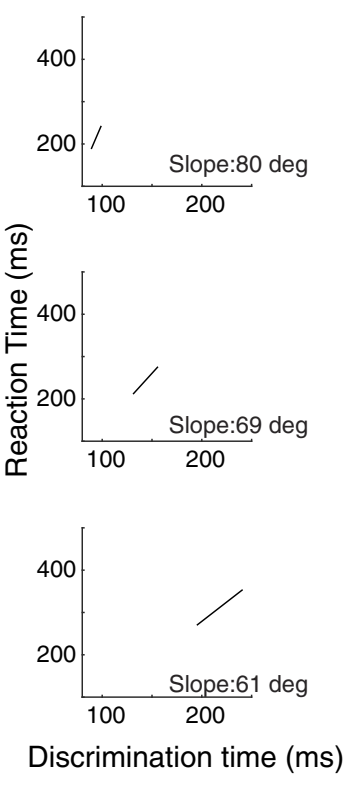

Figure 4. Example results from the same participant (s20) in experiments $1 \mathrm{~b}(\boldsymbol{A}-\boldsymbol{C})$ and $2(\boldsymbol{D}-\boldsymbol{F})$. Same format as Figure 3.

To quantify the influence of the SF of a stimulus on the SLR across our sample, we examined how the prevalence, latency, and magnitude of the SLR change as a function of SF. Prevalence measures the proportion of participants exhibiting an SLR at a particular SF. As shown in Figure 5, top row, SF influenced SLR prevalence in all experiments, with either the lowest SF (experiments $1 \mathrm{~b}$ and 2) or second lowest SF (experiment 1a) being associated with the greatest prevalence, which was sig- nificantly different from the prevalence at the highest SF $\left(\chi^{2}\right.$ test; experiment 1a: $p=0.012, \chi^{2}=6.35$, df $=1$; experiment $1 \mathrm{~b}: p=0.013, \chi^{2}=6.15$, $\mathrm{df}=1$; experiment 2: $p=8.01^{\wedge}-5, \chi^{2}=15.56$, df $=1$ ).

As shown in Figure $2 E$, not all participants exhibited SLRs in each SF condition. This creates an unbalanced design which complicates the analysis of how the characteristics of the SLR vary as a function of SF. To analyze how SLR latency changed as a function of SF in experi- 
A

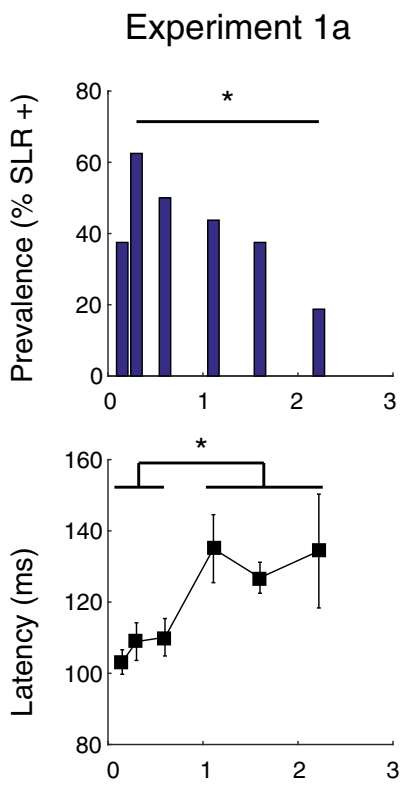

B
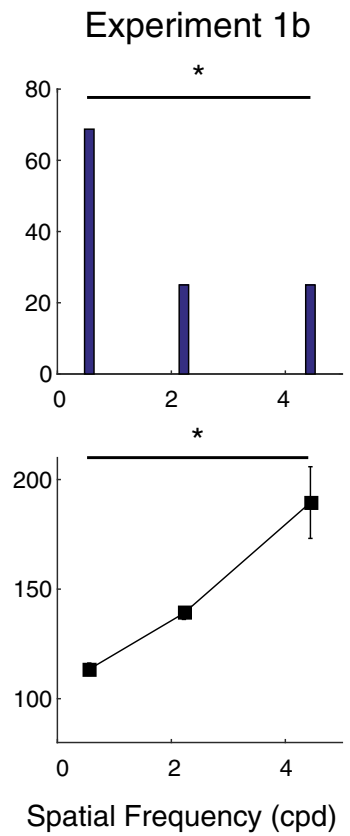

C
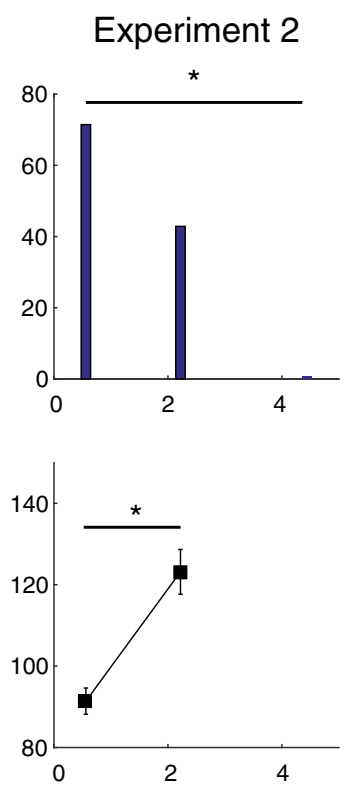

Figure 5. Effect of SF on latency and prevalence of the SLR, across all experiments (Experiments $1 \mathrm{a}, 1 \mathrm{~b}$, and 2 in $\boldsymbol{A}, \boldsymbol{B}$, and $\boldsymbol{C}$ respectively) and participants. In each column, the top row conveys SLR prevalence (the percentage of SLR+ participants across the sample) as a function of SF, and the bottom row conveys SLR latency as a function of SF. Error bars in lower row show SEM. Asterisks depict differences significant at the $p<0.05$ level (for details, see Results).

ment 1a, we split SLR+ observations into "low" $(0.15,0.3$, $0.6 \mathrm{cpd})$ and "high" (1.11, 1.6, $2.22 \mathrm{cpd})$ groups. For each participant, we then took the mean of any latency from an SLR+ observation for each of the low versus high groups, and analyzed results from participants exhibiting at least one SLR+ observation in both of the low and high conditions. For experiments $1 b$ and 2 , we analyzed results from any participant exhibiting an SLR + observation at more than one SF. All experiments revealed an influence of stimulus SF on the latency of the SLR, with lower SFs eliciting shorter-latency SLRs [paired $t$ tests; experiment 1a: $t_{(9)}=-5.37, p=0.00045$ (Fig. $5 A$, bottom panel); experiment 1b: $t_{(5)}=-3.64, p=0.015$ (Fig. $5 B$, bottom panel); experiment 2: $t_{(3)}=-6.13, p=0.0087$ (Fig. 5C, bottom panel)].

We attempted to conduct a similar analysis on the magnitude of the SLR response, but such an analysis was complicated by the increases in SLR latency that we observed for higher SFs. As a consequence, the large volley of EMG activity associated with movement-related activity often overlapped with the SLR interval (for the 0.56 vs $2.22 \mathrm{cpd}$ condition, see Fig. $4 D$ ), obscuring the quantification of EMG recruitment attributable to the SLR. SLRs tended to be easily distinguished at the lower but not higher SFs, as shown both by the vertical banding tied to stimulus onset in trial-by-trial EMG activity, and a distinct initial peak or plateau in the time-series ROC plots (see also Wood et al., 2015). In contrast, time-series ROC plots for higher SF stimuli, regardless of whether they were classified as SLR+ or SLR-, tended to lack a clear initial peak or plateau after discrimination (Fig. $4 E$, second and third rows). The lack of a clear initial peak in the time-series ROC for higher SF stimuli reinforces our concerns about whether an analysis of EMG recruitment during the SLR interval would fairly capture only activity attributable to the SLR, rather than also including movement-related activity on some subset of trials.

Overall, despite our inability to comprehensively quantify SLR magnitude in what we felt was a fair manner uncontaminated by movement-related activity, in all experiments we observed clear trends for lower SF stimuli to elicit shorter-latency and more prevalent SLRs.

\section{Latency and prevalence effects persist in results controlled for RT}

As described in the methods, all peripheral stimuli were perceptually contrast-matched across SFs in an attempt to minimize potential perceptual confounds. Consistent with previous results, although contrast matched, higher SFs elicited longer RTs (Breitmeyer, 1975). Could the dependencies with SF noted in the previous section simply result from the generation of shorter RTs at lower SFs? To address this potential confound, we conducted a within-subject RT matching procedure in which we selected a subset of trials at different SFs with overlapping RTs. In the matching procedure, leftward and rightward reach RT data from low SFs (the 0.6-cpd stimulus in experiment $1 \mathrm{a}$, the 0.56 -cpd stimulus in experiments $1 \mathrm{~b}$ and 2) were matched to leftward and rightward reach high SFs data (the 2.22-cpd stimulus in all experiments), respectively. In the first step of the matching procedure, we matched pairs of trials with identical RTs, without replace- 
ment. We then iteratively identified pairs of trials with RTs within \pm 15 ms of each other, again without replacement, prioritizing the shortest RTs. Once this matching procedure was completed, we only analyzed datasets with $>45$ RT-matched trials.

This procedure yielded distributions with very similar RTs for leftward or rightward reaches across different SFs. The distributions of RTs matched in this way were not significantly different in 31/32 comparisons for experiment 1a, 30/32 comparisons in experiment $1 \mathrm{~b}$, and 26/28 comparisons in experiment 2 ( $t$ test, testing at $p<0.05$, conservatively not correcting for multiple comparisons). Of those few distributions where RTs were found to be different, mean RTs differed by $<8 \mathrm{~ms}$. We then repeated our analyses of the SLR on this subset of trials, closelymatched for RTs.

Figure 6 shows EMG data and time-series ROC plots for a representative participant from each of the three experiments. From these examples, it is clear that the dependency of SLR with SF persists even with closelymatched RTs. For example, a clear SLR evolves after the presentation of a lower SF stimulus in experiments $1 \mathrm{a}, 1 \mathrm{~b}$, and 2, but not at the higher SF. Further, any features of EMG recruitment that appeared to be related to the higher SF stimulus evolved $\sim 30$ ms later than to the low SF stimulus, despite closely matched RTs (Fig. 6G). In these examples, the influence of SF on the earliest directiondependent EMG activity is particularly apparent in the mean EMG and time-series ROC plots shown in the right column of Figure 6, in that the divergence of EMG activity following leftward versus rightward target presentation occurs earlier when the target is composed of low than high SFs (green vs purple traces).

Figure 7 shows SLR prevalence and latency across our sample for RT-matched data. From this figure it is clear that lower SFs continued to elicit more prevalent (Fig. $7 A, C, E$ ) and shorter latency (Fig. $7 B, D, F$ ) SLRs, even when RTs are closely matched. These results reached significance for SLR prevalence in experiment $2(p=$ $0.018, \chi^{2}=5.6, \mathrm{df}=1$ ), and for SLR latency in experiment $1 \mathrm{~b}\left(t\right.$ test; $t_{(15)}=-3.24, p=0.0054$; Fig. $\left.7 D\right)$, and experiment 2 ( $t$ test; $t_{(8)}=-2.65, p=0.029$; Fig. 7F). These analyses confirm that the dependencies of SLR responses properties on the SF of a stimulus are not simply the result of short RTs to stimuli composed of lower SFs.

\section{SLRs, and muscle recruitment more generally, evolve earlier when the arm is already in motion}

We noticed in Figure 5 that SLR latencies were shorter when the arm was already in motion (experiment 2) versus in a stable posture (experiment 1). Recall that experiments $1 \mathrm{~b}$ and 2 were configured so that the retinal location of peripheral targets, and the spatial position of the hand at peripheral target onset, were approximately equal (Fig. $1 A, B)$. Therefore, we investigated the differences in SLR latency to the same stimulus between experiment $1 \mathrm{~b}$ and experiment 2. To do this, we plotted the SLR latency in experiment 2 as a function of that in experiment $1 \mathrm{~b}$. As illustrated in Figure $8 A$, all but one participant response fell below the line of unity, indicating shorter SLR latencies in experiment 2 (paired $t$ test, $t_{(7)}=4.88, p=0.0018$ ). Thus, in those few instances where an SLR was provoked to the same stimulus in both experiments $1 b$ and 2 for the same participant, shorter SLRs were observed when the hand was already in motion ( $22 \pm 13 \mathrm{~ms}, n=8)$. Note that since these values are specifically locked to stimulus onset, this $\sim 20$-ms difference is not due to the lower RT cutoff used in experiment 2.

The inclusion criteria for the analysis shown in Figure 8A is strict, in that a given participant would have to display an SLR to a given SF in both experiment $1 \mathrm{~b}$ and experiment 2. We were also interested in the time at which EMG activity indicated the side of target presentation (or equivalently movement direction, since participants are reaching toward the target) across our entire sample, regardless of whether an SLR was detected or not. One can think of this measure as being akin to time at which mean EMG activity diverges for movements to the right or left; in the case of SLR- observations, EMG recruitment would be related to movement onset rather than target presentation. For consistency with Figure $8 A$, we compared the discrimination times determined by the timeseries ROC analysis for all participants and all stimuli across Experiments $1 \mathrm{~b}$ and 2. As shown in Figure $8 B$, earlier discrimination times persisted in experiment 2 (paired $t$ test, $t_{(41)}=6.25, p=1.9 \mathrm{e}-7$ ). Comparatively, we observed a smaller difference in discrimination time in those observations that lacked an SLR in both the static and dynamic experiment (13 $\pm 15 \mathrm{~ms}$ shorter in experiment $2, n=34)$. Interestingly, as seen in Figure $8 B$, SF also appeared to influence the comparative discrimination time, as the higher SFs clustered closer to the line of unity (repeated measures ANOVA, $F_{(2,26)}=3.76, p=0.037$; Fig. $8 B$, red vs black differences). Thus, when the arm is in motion, information that arises after target presentation gets to the upper limb sooner, driving shorter latency SLRs when that phenomenon is present, and shorterlatency recruitment related to movement onset when the SLR is absent. However, this effect is greatest in the presence of an SLR, and to low SF stimuli.

\section{Paralleling SLRs, on-line reach corrections start earlier for lower SF stimuli}

All participants demonstrated a similar pattern of online corrections (Fig. 9A), wherein the lowest SF evoked the shortest latency corrections. Paralleling SLR responses, the corrective latency systematically increased (one-way repeated measures ANOVA, $F_{(2,26)}=99.78, p=$ 6.34e-13; Fig. $9 B$ ) as a function of SF. We also measured the time it took for a participant to attain the position of the jumped target, and found that this time-to-target measure also increased significantly for higher SFs (one-way repeated measures ANOVA, $F_{(2,26)}=83.36, p=4.91 \mathrm{e}-12$; Fig. 9C). We also quantified aspects of movement kinematics, doing so in a number of ways. First, we calculated the magnitude of the on-line reach correction over the 400 ms after target onset as the area between the mean left and right reach trajectories, and found that this measure both systematically decreased with increasing SF (oneway repeated measures ANOVA, $F_{(2,26)}=437.86, p=$ 
A

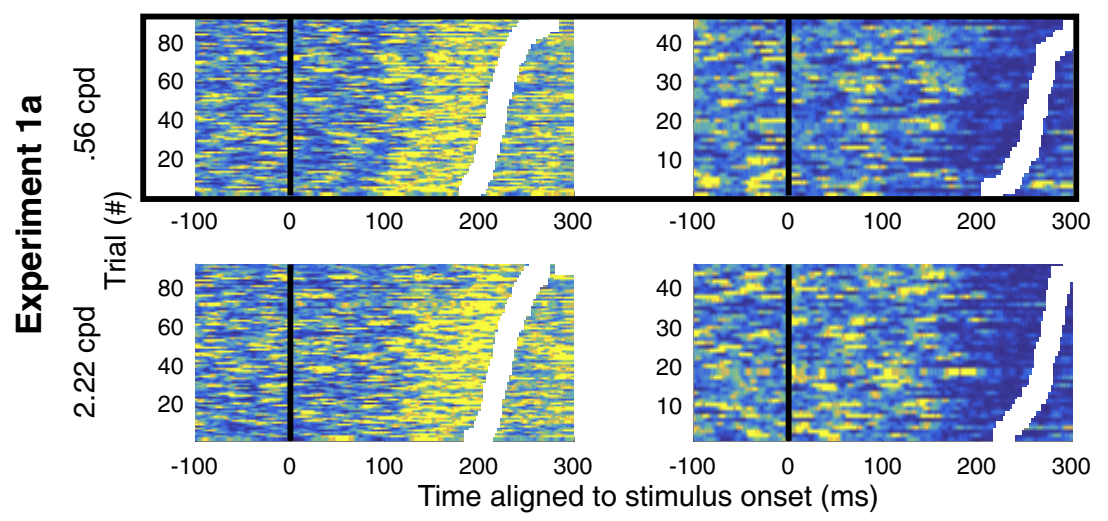

D

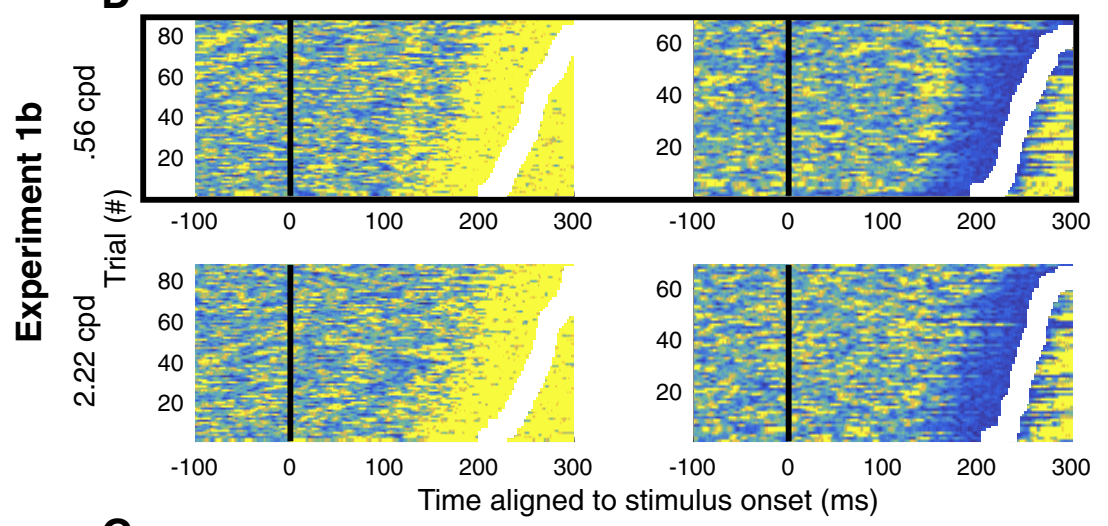

G

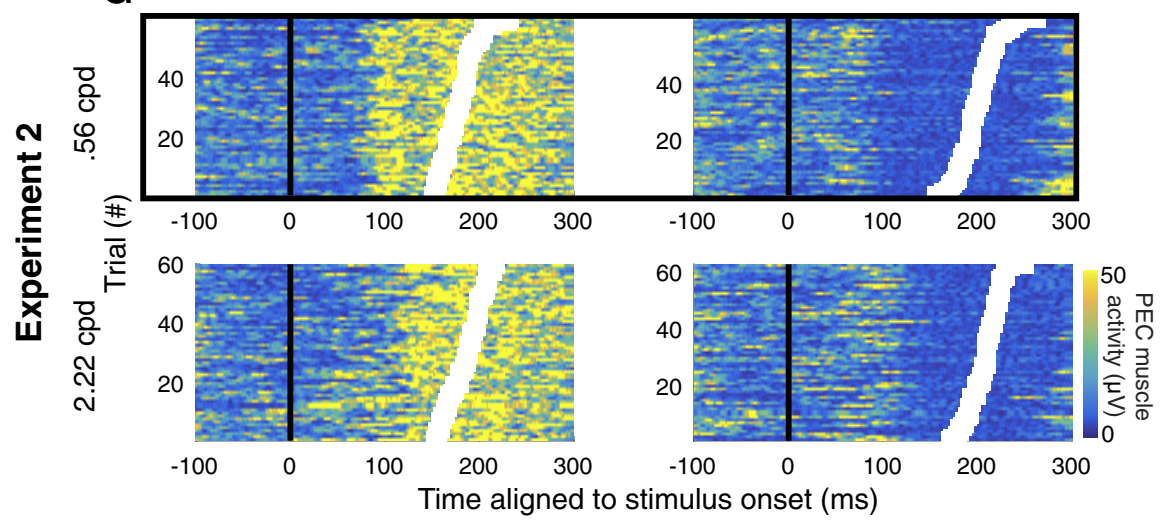

B

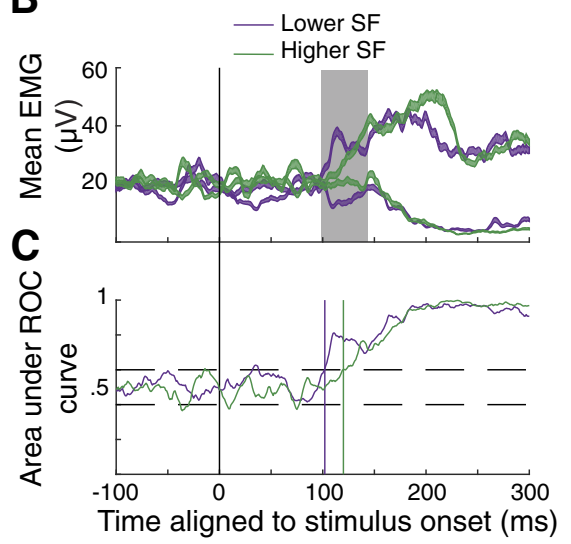

E

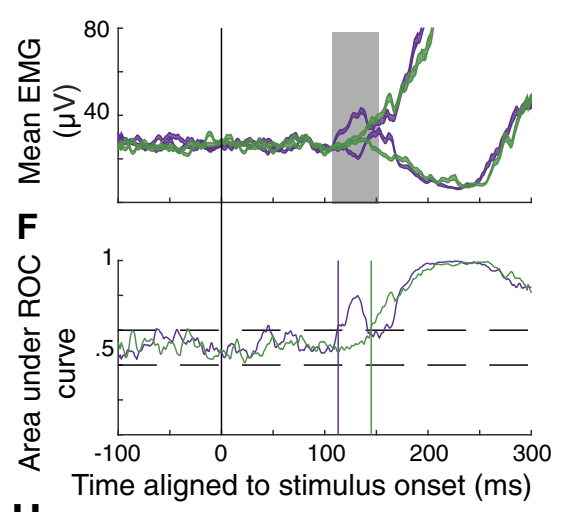

H

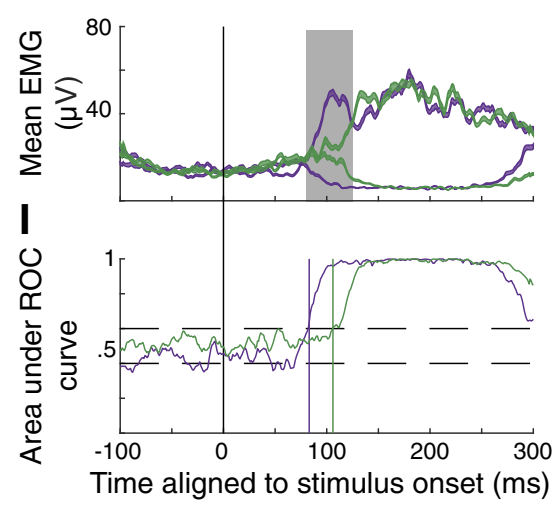

Figure 6. Example results from all experiments for trials matched by RTs. Trial-by-trial EMG activity (A, $\boldsymbol{D}, \boldsymbol{G}$; same format as Fig. 1C), mean EMG activity $(\boldsymbol{B}, \boldsymbol{E}, \boldsymbol{H}$; same format as Fig. $1 D)$, and time-series ROC analysis $(\boldsymbol{C}, \boldsymbol{F}$, $\boldsymbol{I}$; same format as Fig. $1 E)$ for data from experiment 1a $(\boldsymbol{A}-\boldsymbol{C}, \mathbf{s} 14)$, experiment $1 \mathrm{~b}(\boldsymbol{D}-\boldsymbol{F}, \mathbf{s} 30)$, and experiment 2 (G-I, s30). For each experiment, the RTs were matched for individual trials following presentation of a stimulus of a low SF (purple) or high SF (green). The solid boxes around the trial-by-trial plots depict data that exhibits an SLR, even with the reduced number of trials.

9.52e-21; Fig. 9D) and correlated significantly across participants with the time-to-target measure $(R=-0.73, p=$ $2.82 \mathrm{e}-8)$. Second, we also measured the dispersion to the left versus right during the on-line reach correction, and found that this measure did not change systematically across SF (one-way repeated measures ANOVA, $F_{(2,22)}=$ 3.38, $p=0.0526$; Fig. $9 E$ ). Thus, in contrast to previous reports showing that corrections that start earlier tend to proceed more gradually (Oostwoud Wijdenes et al., 2011), varying the SF of a target did not greatly impact the kinematics of the on-line reach correction. Instead, the SF of the jumped target influenced the time at which such corrections are initiated, and correspondingly the time at which the jumped target is reached.

Experiment 2 offers the opportunity to examine SLRs, EMG recruitment more broadly, and the parameters of on-line reach corrections in the same participants. Therefore, we collapsed across conditions to examine a range of responses. We observed that the latency and the magnitude of the on-line corrections were significantly corre- 
A
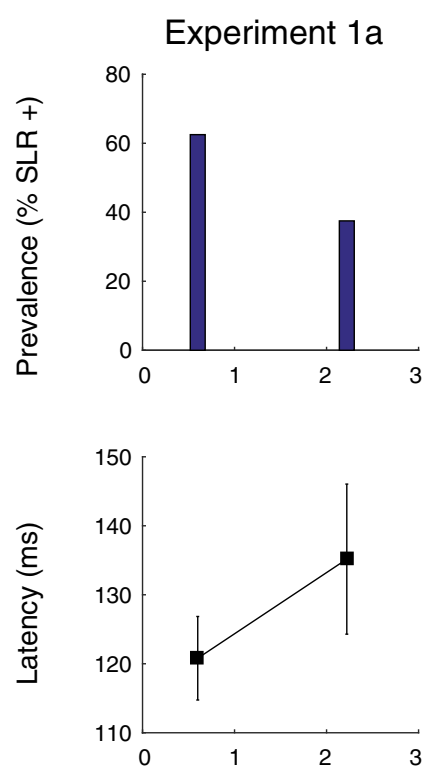

B
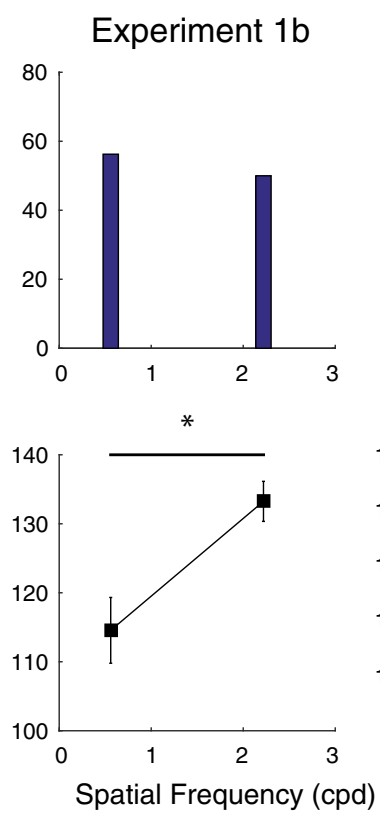

C
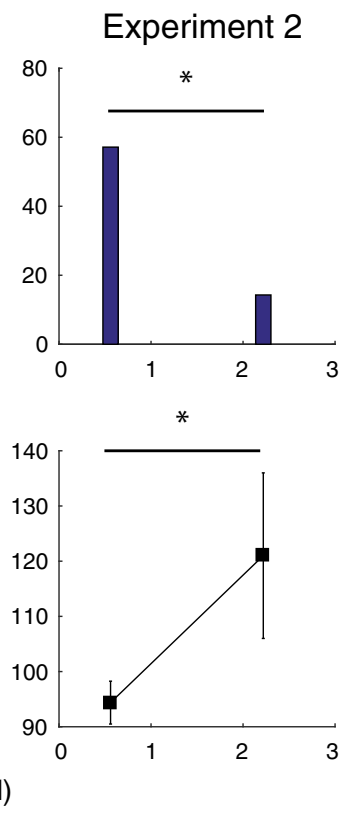

Figure 7. The influence of SF on SLR characteristics persisted for RT-matched trials (data from Experiments 1a, 1b, and 2 shown in $\boldsymbol{A}, \boldsymbol{B}$, and $\boldsymbol{C}$ respectively). Same format as Figure 5, using data matched for RT.

lated (Pearson's correlation, $\left.R^{2}=0.79, p=2.27 \mathrm{e}-15\right)$, indicating that earlier corrections related to larger lateral movement. Furthermore, as would be expected in a muscle contributing to the on-line correction, the latency of EMG discrimination was also significantly correlated with the latency of the reach correction (Pearson's correlation, $\left.R^{2}=0.77, p=6.06 \mathrm{e}-15\right)$, with EMG recruitment preceding kinematic changes by $128 \pm 18 \mathrm{~ms}$ in experiment 2 . As seen in Figure 10, there was also a clear three-way relationship between EMG responses, and the latency and magnitude of on-line reach corrections. Thus, as expected, earlier changes in EMG recruitment were associated with earlier and larger on-line reach corrections.

We also explored the relationship between the presence or absence of SLRs and on-line reach behavior. Firstly, the impression from Figure 10 is that all the data across participants falls along a smooth continuum, regardless of a response being SLR+ or not. Secondly, along this continuum, SLR + observations tended to cluster near the most rapid and largest on-line corrections. To show this, we performed a $k$-means cluster analysis (with $k=3$ ) and found that SLRs predominated in the clusters associated with the earliest and largest corrections [SLR prevalence of $73 \%(11 / 15), 35 \%(5 / 14)$, and $0 \%(0 / 13)$ as clusters increased in latency]. Taken together, earlier EMG responses led to earlier and larger on-line corrections. If evoked, SLRs were associated with earlier and larger on-line corrections.

\section{Discussion}

To interact with our visual world, we are required to transform visual information into motor commands. Here, we systematically investigated which SFs preferentially engender rapid visuomotor responses. We analyzed SLRs on upper limb muscles, as participants performed visually guided reaches toward either stationary targets from a static posture, or targets abruptly displaced while a reaching movement is in mid-flight. For all experiments, we found that low SFs elicited shorter latency and more prevalent SLRs. Paralleling SLRs, on-line corrections started earlier for low SFs, and consequently attained greater magnitudes. From this research, it is clear that a rapid visuomotor pathway preferentially transforms low SF visual information into the earliest phases of muscle recruitment.

\section{A fast visuomotor system prefers lower SFs}

A variety of visual stimulus attributes, including stimulus color, intensity, and SF have also been investigated in other rapid visuomotor responses such as saccadic RTs (Bell et al., 2006; White et al., 2009; Chen et al., 2018) and ocular and manual following responses (Miles et al., 1986; Gellman et al., 1990; Saijo, 2005; Gomi et al., 2006). These results indicate that rapid visuomotor responses rely heavily on the processing of some visual attributes, but not others. Consistent with these findings, we demonstrate that the pathway mediating rapid EMG responses and the latency of on-line corrections are preferentially sensitive to low SFs. Veerman et al. (2008) studied the influence of many stimulus attributes on the latency of on-line corrections and found that the shortest RT responses were generated in response to stimuli defined by contrast, orientation, or size. Importantly, although Veerman et al. (2008) examined reactions to a textured square, the dominant SF of the texture was not provided nor systematically varied. We further this research and show that SLRs are preferentially evoked by low SFs that may be elicited from either a static or dynamic limb position. 
A
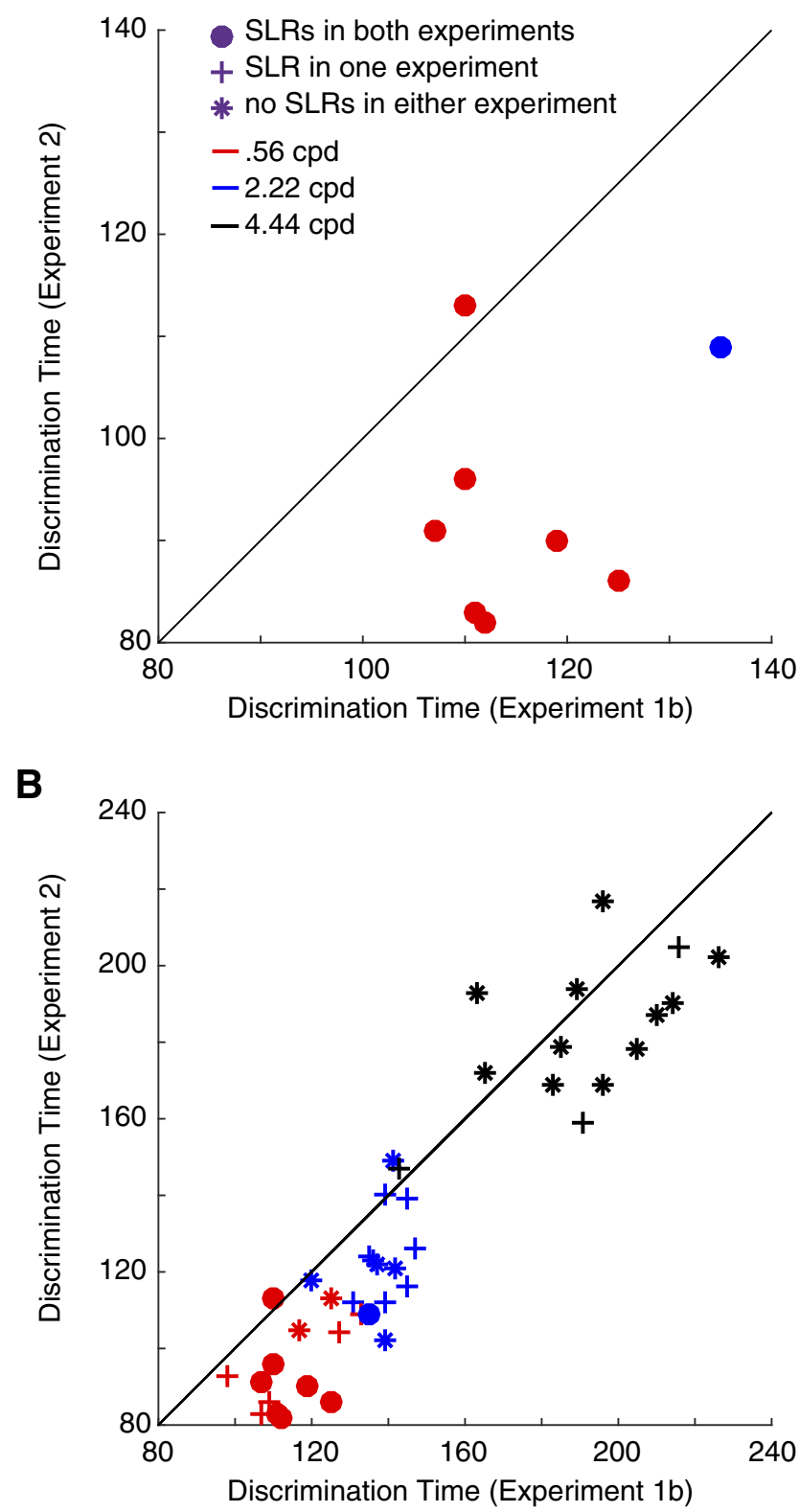

Figure 8. Discrimination times during on-line reach corrections (experiment 2) plotted as a function of discrimination times for reaches initiated from a static posture (experiment 1b). Discrimination times are derived from the time-series ROC analysis. Each data point depicts data from a unique combination of subject and stimulus SF (see color scheme). A, Data from occurrences with an SLR+ observation in both experiment $1 \mathrm{~b}$ and experiment 2. B, Data from all participants, with symbols depicting whether SLRs were observed in both experiments (dots, same data as in $\boldsymbol{A}$, but on a different time scale), whether an SLR was observed in only one experiment (crosses), or whether an SLR was not observed in either experiment (asterisks). Solid diagonal line shows line of unity. The clustering of data below the line of unity means that shorter discrimination times were observed in experiment 2.
When present in experiment 2, SLRs are associated with shorter latency on-line reach corrections (Fig. 10).

We emphasize that we are not claiming that SLRs are solely responsible for on-line reach corrections; after all, muscle recruitment during on-line reach corrections elaborates over hundreds of milliseconds, likely due to a signaling along a host of descending motor pathways (see next section). Instead, our results show remarkable similarities between SLRs and the initiation latency of on-line reach corrections. To put it another way, on-line reach corrections are clearly possible in the absence of SLRs, but such corrections are initiated at a longer latency.

\section{Plausible neural substrates for SLRs and the initial phase of on-line reach corrections}

One potential structure that could serve as a sensorimotor interface for fast visuomotor responses is the superior colliculus (SC), a midbrain structure involved in coordinating the orienting reflex whereby an organism is able to rapidly realign the eyes, head, and upper body toward novel stimuli of interest (for review, see Corneil and Munoz, 2014). There are many similarities between visual responses in the intermediate layers of the SC (SCi) and the SLR. For example, in anti-saccade or antireaching tasks, visual responses in the SCi (Everling et al., 1999), and the SLR on neck (Corneil et al., 2008) or limb muscles (Gu et al., 2016) are tuned to stimulus location. Visual responses present in reach-related SCi neurons also correlate well with upper limb muscle activity (Werner et al., 1997a,b; Stuphorn et al., 1999), with SCi responses preceding SLRs in neck muscles by approximately $\sim 11$ $\mathrm{ms}$, consistent with the efferent lag from the SCi to the neck (Rezvani and Corneil, 2008). Further, visual responses in the SCi (Li and Basso, 2008; Marino et al., 2012), SLRs on the upper limb (Wood et al., 2015), and on-line corrections (Veerman et al., 2008) all evolve at shorter latencies in response to high-contrast visual stimuli, or low SFs (Chen et al., 2018; current results). Indeed, SLRs have been argued to resemble many aspects of express saccades (Corneil et al., 2004), when visual responses in the SCi become strong enough to drive recruitment in the motor periphery. Consistent with this perspective, express saccades have also been argued to occur at long latencies to suboptimal stimuli (Bell et al., 2006), which has been observed in SLRs toward low contrast (Wood et al., 2015), or higher SF (Fig. 5) stimuli.

In contrast to our results, Chen et al. (2018) reported that some SCi neurons exhibited the greatest magnitude responses to higher SF stimuli in the 2- to 4-cpd range, even if such responses started later than those elicited by lower SFs. Why then did we not observe more SLRs at such SFs? We speculate that there are two related issues. First, Chen et al. (2018) reported on the peak sensitivities of individual neurons, and while there were indeed some neurons that preferred stimuli between 2-4 cpd, such neurons were outnumbered by those neurons preferring lower SFs (Chen et al., 2018, their Fig. 3). If our hypothesis about involvement of the SCi in the limb SLR is correct, then it seems reasonable that the SLR would be produced by the population of responding SCi neurons, which as a 
A

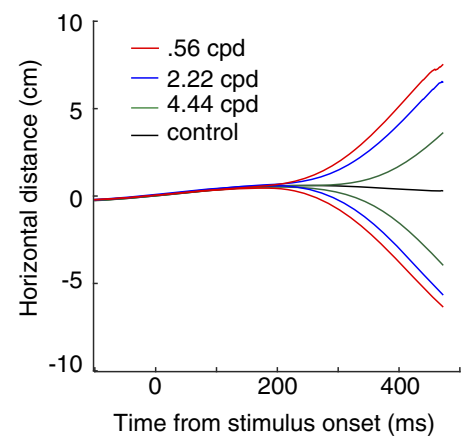

B

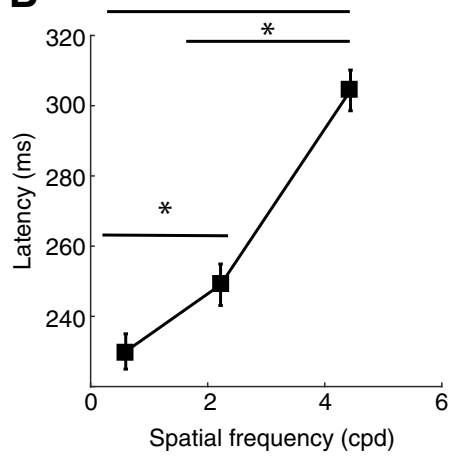

C

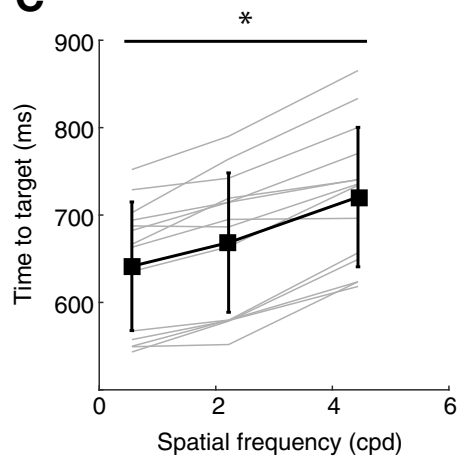

$\mathbf{E}$

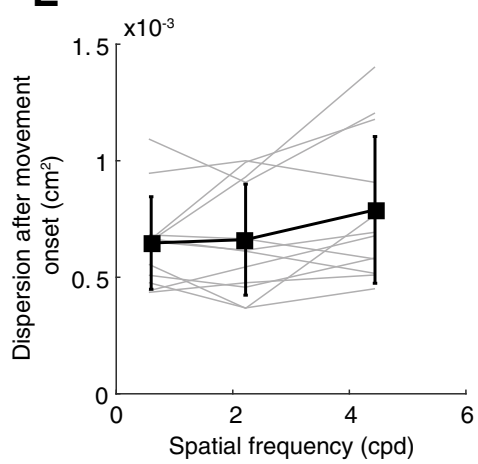

Figure 9. Characteristics of on-line reach corrections to different SFs in experiment 2. $\boldsymbol{A}$, Results from a sample participant (s20), depicting the mean lateral distance for on-line reach corrections to displaced stimuli composed of different SFs (colored lines), or to the control condition where the reach target was not displaced (black line). B, Initiation latency of the on-line reach correction as a function of SF, across the sample. The latency was derived from a time-series ROC analysis of lateral distance for left versus right displaced stimuli. $\boldsymbol{C}$, The time to target, measured as the time from target jump to the time at which the hand attains the jumped target, also varies as a function of SF. $\boldsymbol{D}$, The SF of the jumped target influenced magnitude of the on-line reach correction over the 400 ms after the target jump (measured as the area between the left and right trajectories over this 400-ms interval, normalized to the maximum within each participant). $\boldsymbol{E}$, In contrast, the dispersion of the horizontal component between left and right on-line reach correction, measured from the initiation of the correction to the point at which the vertical component has progressed a further $4 \mathrm{~cm}$, did not vary with SF (in $\boldsymbol{E}$, data from two participants were excluded since many corrections were initiated $<4 \mathrm{~cm}$ along the $\mathrm{y}$ component from the central target in at least one condition). Error bars represent SEM. Asterisks depict differences significant at the p 0.05 level (for details, see Results).

whole would prefer lower SFs. Second, and likely more critically, Chen et al. (2018) reported that the low-pass filtering of SFs exhibited by SCi neurons got stronger for neurons with more eccentric response fields (Chen et al., 2018, their Fig. 6). We presented our stimuli at eccentricities of $\sim 8^{\circ}$ or $9^{\circ}$ where the proportion of neurons preferring SFs $\leq 1 \mathrm{cpd}$ is even greater, and not at foveal locations where neurons preferring higher SFs are more plentiful. An interesting future direction would be to vary the spatial eccentricity of stimuli eliciting the SLR, as the Chen results would predict that SLRs should be driven by stimuli composed of higher SFs that are presented closer to the fovea.

Our position is that SLRs, and by extension, the initial phase of on-line reach corrections, are mediated by visual responses in the SCi. While this idea is consistent with previous ideas of how the SCi may be involved in rapid visuomotor responses (Day and Brown, 2001), in general it is thought that on-line reach corrections are driven through either parietal or frontal sources (for review, see Gaveau et al., 2014; Archambault et al., 2015). Although many of the stimulus preferences for SLRs and on-line reach corrections resemble those reported in the $\mathrm{SCi}$, we are mindful that other areas in the parietal and frontal cortices implicated in on-line reach control also exhibit visually-aligned response transients (Riehle, 1991; Cisek and Kalaska, 2005), although the relationship between such transients and various stimulus features remain to be investigated. The general similarities that we are observing between SLRs and the early phase of on-line reach corrections suggests that a subcortical route involving the SCi may provide the earliest drive to the motor periphery. Subsequent, more contextually-guided portions of the on-line correction may rely on corticospinal inputs that incorporate processing in frontal and parietal cortices.

\section{Expedited visuomotor responses when the limb is already in motion}

Experiments $1 \mathrm{~b}$ and 2 were designed to compare SLRs in situations where stimulus presentation occurred when the limb was either stable or already in motion. We found that SLRs, and the earliest divergence of EMG activity, following stimulus onset evolved substantially earlier ( $\sim 20-30 \mathrm{~ms})$ when the limb was already in motion. This 


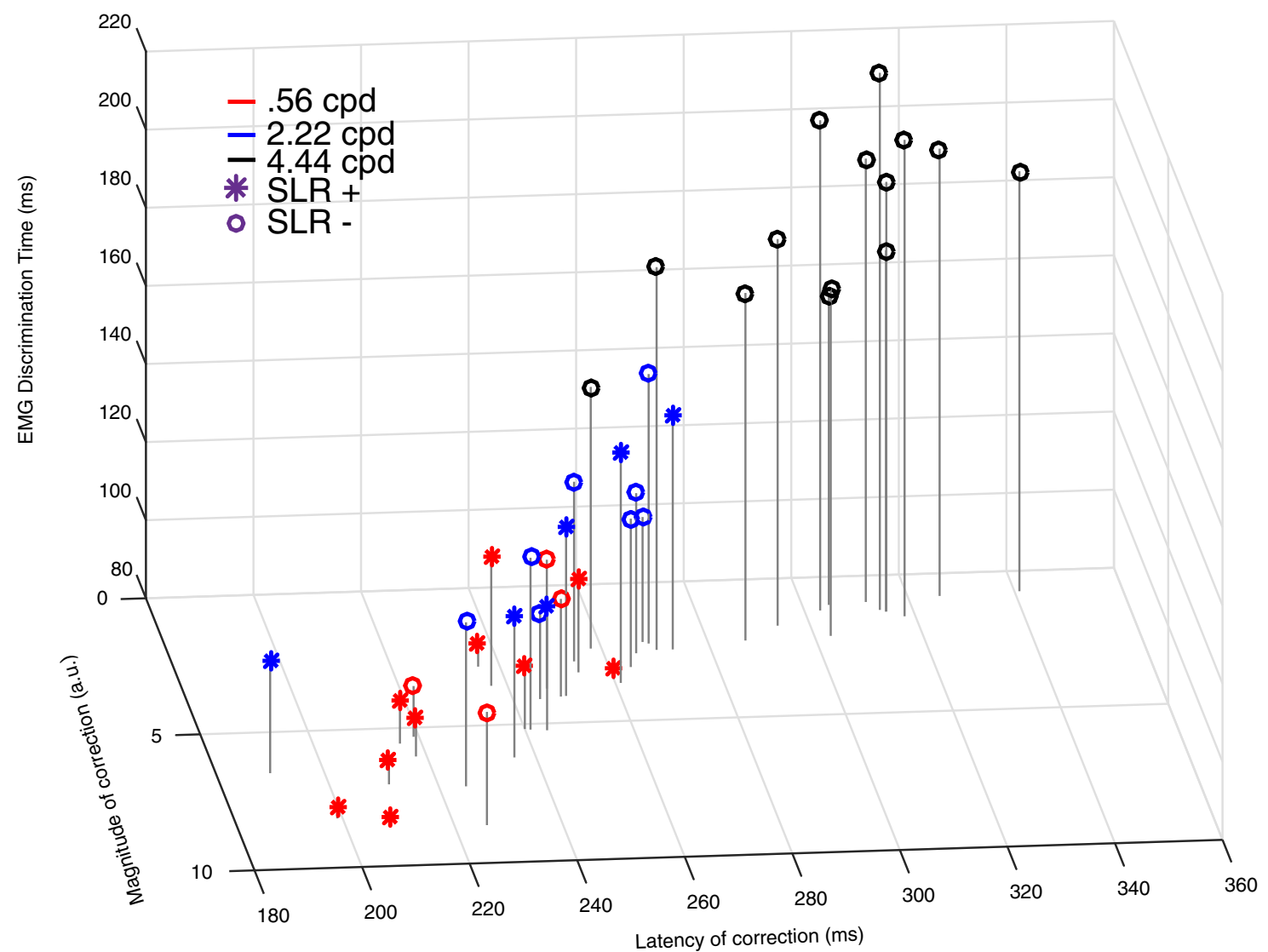

Figure 10. Three-way relationship between EMG discrimination, and the latency and magnitude of the on-line reach correction (as measured in Fig. 9D). Each point represents data from a single participant, color-coded by SF, with the symbol indicating whether an SLR was observed (asterisks) or not (circles).

observation relates to contemporary debates about expedited RTs during movements initiated from a dynamic position (Smeets et al., 2016), and are consistent with expedited responses occurring when a postural control policy has been disengaged (Scott and Cluff, 2016). What is interesting is to consider where in the pathway rapidly transforming vision into actions the delay in activity aligned to stimulus onset is delayed by 20-30 ms when movements are beginning from a stable posture, and why such a delay depends on SF. It would appear that any such delay would have to be imposed downstream from the SCi, given that visual response latencies in the SCi approach the minimal afferent delay.

\section{Methodological considerations}

Our experimental configuration limited the upper range of SFs we could present without aliasing (2.22 and 4.44 cpd in experiments 1 and 2, respectively). We do not view this as a limitation, as SLRs were rarely generated in response to a 4.44-cpd stimulus. Further, any SLRs that were generated to the 4.44-cpd stimulus did so at a latency that overlapped with movement-related activity. In this vein, the potential overlap between the SLR and movement-related activity complicated analysis of SLR magnitude. Future experiments should investigate magnitude in a paradigm which separates SLR and movement related activity, where immediate reaching is not required.
Interestingly, at the lower range of SFs in experiment 1a, we observed that the 0.15 -cpd stimulus elicited the shortest-latency but not necessarily the most prevalent SLR, highlighting how these measures can be dissociated.

We went to some lengths to ensure the trends we observed in SLR latency and prevalence across SFs were not confounded by RT, implementing a contrast-matching procedure and reanalyzing SLRs after matching RTs. Our contrast-matching procedure also ensured that our observed effects were due to the SF of the targets, as opposed to perceived contrast. However, the on-line correction $\mathrm{RTs}$ we observed were longer than those reported previously (e.g., 125 ms in Day and Lyon, 2000). We surmise that there are a few reasons for this discrepancy. Firstly, we observed single-trial examples with RTs below $200 \mathrm{~ms}$ (Fig. 2A), although we reported the mean correction latencies. Secondly, we excluded shorter-latency RTs to prevent overlap with movement-related activity. Most importantly, stimuli defined by texture are known to elicit longer-RT corrections (Veerman et al., 2008).

Finally, SLR prevalence never exceeded $75 \%$, meaning that stimuli did not always elicit an SLR (see also Pruszynski et al., 2010; Wood et al., 2015; Gu et al., 2016). The failure to observe an SLR may be due to participant idiosyncrasies, or to the possibility that the SLR distributes to deeper layers of fatigue-resistant 
muscle (Pruszynski et al., 2010). As mentioned above, Gabor patches may also be a suboptimal stimulus for the SLR. Recall also that our classification criteria relied on a median-split analysis. This is a conservative approach that requires a substantial number of trials and considerable variance in RTs.

\section{Conclusions}

The work presented here builds on a stream of findings that emphasize the importance of stimulus attributes on visually-evoked responses in premotor areas and the motor periphery, showing that the earliest component of a rapid visuomotor response is preferentially elicited by low SF stimuli. The data from experiment 2 are the first to directly link the phenomenon of SLRs to on-line reach corrections in the same task and in the same participants. This link will help further characterizations of the substrates underlying rapid visuomotor responses, as the SLRs can be readily elicited on a trial-by-trial basis while participants are in a stable posture. Detailing the stimulus attributes which best elicit rapid visuomotor responses will help identify plausible underlying substrates and will aid the study of such responses across the lifespan and in clinical populations.

\section{References}

Archambault PS, Ferrari-Toniolo S, Caminiti R, Battaglia-Mayer A (2015) Visually-guided correction of hand reaching movements: the neurophysiological bases in the cerebral cortex. Vision Res 110:244-256.

Atsma J, Maij F, Gu C, Medendorp WP, Corneil BD (2018) Active braking of whole-arm reaching movements provides single-trial neuromuscular measures of movement cancellation. $J$ Neurosci 38:4367-4382.

Bell AH, Meredith MA, Van Opstal AJ, Munoz DP (2006) Stimulus intensity modifies saccadic reaction time and visual response latency in the superior colliculus. Exp Brain Res 174:53-59.

Brainard DH (1997) The psychophysics toolbox. Spat Vis 10:433436.

Breitmeyer BG (1975) Simple reaction time as a measure of the temporal response properties of transient and sustained channels. Vision Res 15:1411-1412.

Chen CY, Sonnenberg L, Weller S, Witschel T, Hafed ZM (2018) Spatial frequency sensitivity in macaque midbrain. Nat Commun 9:2852.

Cisek P, Kalaska JF (2005) Neural correlates of reaching decisions in dorsal premotor cortex: specification of multiple direction choices and final selection of action. Neuron 45:801-814.

Corneil BD, Munoz DP (2014) Overt responses during covert orienting. Neuron 82:1230-1243.

Corneil BD, Olivier E, Munoz DP (2004) Visual responses on neck muscles reveal selective gating that prevents express saccades. Neuron 42:831-841.

Corneil BD, Munoz DP, Chapman BB, Admans T, Cushing SL (2008) Neuromuscular consequences of reflexive covert orienting. Nat Neurosci 11:13-15.

Davidson M (1968) Perturbation approach to spatial brightness interaction in human vision. J Opt Soc Am 58:1300-1308.

Day BL, Lyon IN (2000) Voluntary modification of automatic arm movements evoked by motion of a visual target. Exp Brain Res 130:159-168.

Day BL, Brown P (2001) Evidence for subcortical involvement in the visual control of human reaching. Brain 124:1832-1840.

Everling S, Dorris MC, Klein RM, Munoz DP (1999) Role of primate superior colliculus in preparation and execution of anti-saccades and pro-saccades. J Neurosci 19:2740-2754.
Gaveau V, Pisella L, Priot A-E, Fukui T, Rossetti Y, Pélisson D, Prablanc C (2014) Automatic online control of motor adjustments in reaching and grasping. Neuropsychologia 55:25-40.

Gellman RS, Carl JR, Miles FA (1990) Short latency ocular-following responses in man. Vis Neurosci 5:107-122.

Gomi H, Abekawa N, Nishida S (2006) Spatiotemporal tuning of rapid interactions between visual-motion analysis and reaching movement. J Neurosci 26:5301-5308.

Goonetilleke SC, Katz L, Wood DK, Gu C, Huk AC, Corneil BD (2015) Cross-species comparison of anticipatory and stimulus-driven neck muscle activity well before saccadic gaze shifts in humans and nonhuman primates. J Neurophysiol 114:902-913.

Gu C, Wood DK, Gribble PL, Corneil BD (2016) A trial-by-trial window into sensorimotor transformations in the human motor periphery. $J$ Neurosci 36:8273-8282.

Gu C, Pruszynski JA, Gribble PL, Corneil BD (2018) Done in 100 ms: path-dependent visuomotor transformation in the human upper limb. J Neurophysiol 119:1319-1328.

Li X, Basso MA (2008) Preparing to move increases the sensitivity of superior colliculus neurons. J Neurosci 28:4561-4577.

Marino RA, Levy R, Boehnke S, White BJ, Itti L, Munoz DP (2012) Linking visual response properties in the superior colliculus to saccade behavior. Eur J Neurosci 35:1738-1752.

Miles FA, Kawano K, Optican LM (1986) Short-latency ocular following responses of monkey. I. Dependence on temporospatial properties of visual input. J Neurophysiol 56:1321-1354.

Oostwoud Wijdenes L, Brenner E, Smeets JBJ (2011) Fast and fine-tuned corrections when the target of a hand movement is displaced. Exp Brain Res 214:453-462.

Pelli DG (1997) The VideoToolbox software for visual psychophysics: transforming numbers into movies. Spat Vis 10:437-442.

Pruszynski AJ, Kurtzer I, Scott SH (2008) Rapid motor responses are appropriately tuned to the metrics of a visuospatial task. J Neurophysiol 100:224-238.

Pruszynski AJ, King GL, Boisse L, Scott SH, Randall Flanagan J, Munoz DP (2010) Stimulus-locked responses on human arm muscles reveal a rapid neural pathway linking visual input to arm motor output. Eur J Neurosci 32:1049-1057.

Rezvani S, Corneil BD (2008) Recruitment of a head-turning synergy by low-frequency activity in the primate superior colliculus. $J$ Neurophysiol 100:397-411.

Riehle A (1991) Visually induced signal-locked neuronal activity changes in precentral motor areas of the monkey: hierarchical progression of signal processing. Brain Res 540:131-137.

Saijo N (2005) Large-field visual motion directly induces an involuntary rapid manual following response. J Neurosci 25:49414951.

Scott SH (1999) Apparatus for measuring and perturbing shoulder and elbow joint positions and torques during reaching. J Neurosci Methods 89:119-127.

Scott SH, Cluff T (2016) Online corrections are faster because movement initiation must disengage postural control. Motor Control 20:162-170.

Smeets JBJ, Wijdenes LO, Brenner E (2016) Movement adjustments have short latencies because there is no need to detect anything. Motor Control 20:137-148.

Soechting JF, Lacquaniti F (1983) Modification of trajectory of a pointing movement in response to a change in target location. $J$ Neurophysiol 49:548-564.

Stuphorn V, Hoffmann KP, Miller LE (1999) Correlation of primate superior colliculus and reticular formation discharge with proximal limb muscle activity. J Neurophysiol 81:1978-1982.

Veerman MM, Brenner E, Smeets JBJ (2008) The latency for correcting a movement depends on the visual attribute that defines the target. Exp Brain Res 187:219-228.

Werner W, Dannenberg S, Hoffmann K-P (1997a) Arm-movementrelated neurons in the primate superior colliculus and underlying 
reticular formation: comparison of neuronal activity with EMGs of muscles of the shoulder, arm and trunk during reaching. Exp Brain Res 115:191-205.

Werner W, Hoffmann K-P, Dannenberg S (1997b) Anatomical distribution of arm-movement-related neurons in the primate superior colliculus and underlying reticular formation in comparison with visual and saccadic cells. Exp Brain Res 115:206-216.
White BJ, Boehnke SE, Marino RA, Itti L, Munoz DP (2009) Colorrelated signals in the primate superior colliculus. J Neurosci 29: 12159-12166.

Wood DK, Gu C, Corneil BD, Gribble PL, Goodale MA (2015) Transient visual responses reset the phase of low-frequency oscillations in the skeletomotor periphery. Eur J Neurosci 42: 1919-1932. 